Math. Model. Nat. Phenom.

Vol. 9, No. 4, 2014, pp. 171-203

DOI: $10.1051 / \mathrm{mmnp} / 20149411$

\title{
Optimal Control in Coupled Within-host and Between-host Models
}

\author{
E. Numfor ${ }^{1}$, S. Bhattacharya ${ }^{2}$, S. Lenhart ${ }^{1}{ }^{*}$, M. Martcheva ${ }^{2}$ \\ ${ }^{1}$ Department of Mathematics, University of Tennessee, TN 37996, USA \\ ${ }^{2}$ Department of Mathematics, University of Florida, FL 32611, USA
}

\begin{abstract}
We formulate an immuno-epidemiological model of coupled "within-host" model of ODEs and "between-host" model of ODE and PDE, using the Human Immunodeficiency Virus (HIV) for illustration. Existence and uniqueness of solution to the "between-host" model is established, and an explicit expression for the basic reproduction number of the "between-host" model derived. Stability of disease-free and endemic equilibria is investigated. An optimal control problem with drug-treatment control on the within-host system is formulated and analyzed; these results are novel for optimal control of ODEs linked with such first order PDEs. Numerical simulations based on the forward-backward sweep method are obtained.
\end{abstract}

Keywords and phrases: within-host dynamics, between-host dynamics, equilibria, stability, optimal control

Mathematics Subject Classification: 34D20, 35L02, 49K15, 49K20

\section{Introduction}

There is continuous threat of outbreak of infectious disease despite ongoing advancements in drug therapies and vaccines [27]. Thus, it is necessary to develop better ways of understanding the spread of disease. To this effect, immunological and epidemiological models have been proposed with the aim of controlling the outbreak of infectious diseases.

Mathematical immunology is concerned with the study of disease dynamics in an infected host, where an infectious agent is spread from cell to cell within one patient [27]. The study of the interaction between a pathogen and the immune system gives an insight into the mechanism of disease proliferation. In mathematical epidemiology, the spread of disease in a population of hosts is examined with the goal of examining and tracing factors that contribute to the propagation of pathogens [27]. Epidemiological or between-host models are often structured to capture discrete immune status, such as susceptible, exposed, infectious, recovered (immune), vaccinated, time-since-infection to account for variable infectivity (pathogen load) and time-since-recovery to account for gradual loss of immunity. However, most epidemiological models ignore pathogen load and dependence of transmissibility on pathogen load, and detailed account of the immune status during infection [41].

We will investigate linking within-host models with epidemiological models, and as our motivating scenario, we use the human immunodeficiency virus (HIV), which is a retrovirus. In the future, we

\footnotetext{
${ }^{*}$ Corresponding author. E-mail: lenhart@math.utk.edu
} 
shall consider other scenarios such as Johne's disease and Toxoplasma gondii, but we concentrate on HIV for this introduction to our approach. HIV is generally a slow but progressive disease in which the virus is present throughout the body at all stages of the disease, and it is transmitted from one person to another through specific body fluids such as blood, semen, genital fluids, and breast milk. The life cycle of HIV infection consists of six stages; namely, binding and fusion, reverse transcription, integration, transcription, assembly and budding. Several mathematical epidemiology models of HIV [30,33,35,36,47] and mathematical immunology models of HIV [37,44] have been formulated and analyzed.

The two key features in infectious diseases are the transmission between hosts and the immunological process at the individual host level. Understanding how the two features influence each other can be assisted through modeling. Linking components of the immune system with the compartments of the epidemic model leads to a two-scale model. Much of the work on such "linked" models deal with the two levels separately, making "decoupling" assumptions [1].

Despite advancements made with the study of epidemiological, within-host and immunological models, the outbreak of some diseases cannot still be predicted. This dilemma may be attributed to the fact that most modeling approaches are either restricted to epidemiological or immunological formulations, while making decoupling assumptions [27]. Current research focuses on the comprehensive modeling approach, called immuno-epidemiological modeling, which investigates the influence of population immunity on epidemiological patterns, translates individual characteristics such as immune status and pathogen load to population level and traces their epidemiological significance $[12,29,41]$. Several immuno-epidemiological models have been used to study the relationship between transmission and virulence $[5,16,17,21-23]$. Some of these models deal with the two processes separately by making decoupling assumptions. Gilchrist and Sasaki [22] used the nested approach to model host-parasite coevolution in which the within-host model is independent of the between-host but the between-host model is expressed in terms of dependent variables of the within-host model. Also, Feng et al. [16] investigated a coupled within-host and betweenhost model of Toxoplasma gondii linked via the environment.

Our goals are to use a within-host model coupled with epidemiology model to capture the impact on the epidemic of giving treatment to individuals, and investigate mathematically such a coupled ODE/PDE system (well-posedness and optimal control).

Our general approach in immuno-epidemiological modeling involves three steps. The first step involves formulating a within-host model within an infected host. Secondly, construct an epidemiological model to describe the dynamics of host birth and death rates, and transmission of infection within the host population. Finally, nest the within-host model within the epidemiological model by linking the dynamics of the within-host model to the additional host mortality, recovery and transmission rates of the infection. The within-host and between-host models could be linked via a structural variable and through coefficients. In the latter case, coefficients of the epidemiological model are expressed as functions of the dependent variables of the within-host model. For example, transmission rate is proportional to within-host viral load and disease-induced death rate is proportional to parasite load and immune response, while in the former case, the independent variable of the within-host model is the age-since-infection variable of the between-host model $[23,41]$.

This work will have the first results on formulating this two-scale model in a careful mathematical framework and the first results on optimal control of such a model. We emphasize the novelty of mathematical results, as well as the importance of the epidemiological and immunological results. To curtail the proliferation of free virus at the within-host level, we introduce two functions, representing transmission and virion production suppressing drugs. Our goal is to use optimal control techniques in the coupled model to minimize free virus at the within-host level and infectious individuals at the population level, while minimizing the cost of implementing the controls (this may include toxicity effects). Optimal control of first-order partial differential equations is done differently than optimal control of parabolic PDEs due to the lack of regularity of solutions to the first-order PDEs. The steps in justifying the optimal control results are quite different and we use Ekeland's Principle [13] to get the existence of an optimal control. 
In section 2, we present our within-host and between-host models. The within-host model is independent of the between-host model, but the between-host model is linked to the within-host via coefficients and a structural variable. In section 2.1, we prove the boundedness of state solutions to the within-host model, and existence and uniqueness of solutions to the between-host model is established. In section 2.2, an explicit expression for the basic reproduction number of the epidemiological model is derived, steady solutions calculated and stability analysis of equilibrium points is studied. We formulate and analyze an optimal control problem in section 3, and carry out numerical simulations in section 4.

\section{Within-host and Between-host Models}

In this section, we formulate a simple within-host model of HIV and a between-host model of HIV with age structure. In the within-host model, the independent variable is the time-since-infection $\tau$ and for the between-host model, the independent variables are chronological time $t$ and age-since-infection $\tau$. Our within-host model is given by the following system of ordinary differential equations:

$$
\begin{aligned}
& \frac{d x}{d \tau}=r-\beta_{1} V(\tau) x(\tau)-\mu x(\tau) \\
& \frac{d y}{d \tau}=\beta_{1} V(\tau) x(\tau)-d_{1} y(\tau) \\
& \frac{d V}{d \tau}=\nu_{1} d_{1} y(\tau)-\left(\delta_{1}+s_{1}\right) V(\tau)-\hat{\beta}_{1} V(\tau) x(\tau)
\end{aligned}
$$

with initial conditions

$$
x(0)=x^{0}, \quad y(0)=y^{0} \quad \text { and } \quad V(0)=V^{0},
$$

where $x$ is the number of healthy cells (uninfected $C D 4^{+} \mathrm{T}$ cells), $y$ is the density of infected $C D 4^{+} \mathrm{T}$ cells, $V$ is the density of free (infectious) virus, $r$ is the recruitment rate of healthy cells, $\mu$ is the death rate of healthy cells, $d_{1}$ is the death rate of infected cells, $\beta_{1}$ is the transmission rate, $\hat{\beta}_{1}$ is the binding rate of free virus to uninfected $C D 4^{+} \mathrm{T}$ cells, $\nu_{1}$ is the number of virions produced at bursting, $\delta_{1}$ is the death rate of virus, and $s_{1}$ is the shedding rate of virus. See Table 1 for a summary of parameters and units of the within-host model.

Table 1: Within-Host Model Parameters

\begin{tabular}{lll}
\hline Quantity & Description & Units \\
\hline$x$ & Density of healthy CD $4^{+}$T-cells & cell $/ \mathrm{mm}^{3}$ \\
$y$ & Density of infected CD4 ${ }^{+}$T-cells & cell $/ \mathrm{mm}^{3}$ \\
$V$ & Density of free virus & virion $/ \mathrm{mm}^{3}$ \\
$\tau$ & Time since start of infection & days \\
$r$ & Source term for healthy cells $\left(\mathrm{CD} 4^{+}\right.$T-cells $)$ & cell $\mathrm{mm}^{-3} \mathrm{day}^{-1}$ \\
$\mu$ & Natural death rate of healthy cells & day $^{-1}$ \\
$\beta_{1}$ & T cells infection rate by virus & $\mathrm{mm}^{3}$ virion $^{-1}$ day $^{-1}$ \\
$\hat{\beta}_{1}$ & Binding rate of free virus to uninfected & $\mathrm{mm}^{3}$ cell $^{-1}$ day $^{-1}$ \\
& $C D 4^{+}$T cells & \\
$d_{1}$ & Death rate of infected cells & day \\
$\nu_{1}$ & Virion production rate & virion cell \\
$\delta_{1}$ & Death rate of free virus & day \\
$s_{1}$ & Shedding rate of free virus & day \\
\hline
\end{tabular}

Our between-host SI (susceptible, infected) model assumes that the infected class is related to the within-host behavior of a particular individual, and individuals in this class are structured by both chronological time $\mathrm{t}$ and age of infection (age-since-infection), $\tau$. Thus, our between-host model is: 


$$
\begin{aligned}
\frac{d S}{d t} & =\Lambda-\frac{S(t)}{N(t)} \int_{0}^{A} c_{1} s_{1} V(\tau) i(\tau, t) d \tau-m_{0} S(t) \quad \text { in }(0, T) \\
\frac{\partial i(\tau, t)}{\partial t}+\frac{\partial i(\tau, t)}{\partial \tau} & =-m(V(\tau)) i(\tau, t) \quad \text { in }(0, T) \times(0, A) \\
i(0, t) & =\frac{S(t)}{N(t)} \int_{0}^{A} c_{1} s_{1} V(\tau) i(\tau, t) d \tau, \quad \text { for } \quad t \in(0, T) \\
S(0) & =S_{0}, \quad i(\tau, 0)=i^{0}(\tau) \quad \text { for } \quad \tau \in(0, A), t=0
\end{aligned}
$$

where $\mathrm{S}(\mathrm{t})$ is the number of susceptible individuals at time $t, i(\tau, t)$ is the density of infected individuals at time $t$ and age-since-infection $\tau, m(V(\tau))$ is the death rate of infected hosts (a function of viral load), $\Lambda$ is the recruitment rate of susceptible individuals, and $m_{0}$ is the death rate of susceptible individuals. The transmission rate is assumed to be proportional to the viral load of the infected individuals, calculated by integrating with respect to $\tau, \int_{0}^{A} c_{1} s_{1} V(\tau) i(\tau, t) d \tau$, where $c_{1}$ is the contact rate between susceptible and infected individuals. Thus, the new infectious process of the population at time $t$, denoted by $i(0, t)$, depends on the age distribution of the population at time $t$, as determined by the integral of $i(\tau, t)$ over all ages, weighted with the specific transmission rate $\beta(\tau)=c_{1} s_{1} V(\tau)$. The number of susceptible and infectious individuals in the population at time $t=0$ are given by $S(0)=S_{0}>0$ and $i(\tau, 0)=i^{0}(\tau)$, respectively. Thus, $i(\tau, 0)$ is the initial age distribution of infectious individuals in the population, with $i^{0}$ being a known nonnegative function of age-since-infection, $\tau$.

Table 2: Between-Host Model Parameters

\begin{tabular}{lll}
\hline Quantity & Description & Units \\
\hline$\tau$ & Age-since-infection & days \\
$t$ & Chronological time & years \\
$A$ & Maximal age-since-infection & years \\
$S(t)$ & Susceptible individuals at time $t$ & humans \\
$i(\tau, t)$ & Infected individuals of age $\tau$ and time $t$ & humans \\
$S(0)$ & Initial population of susceptible individuals & humans \\
$i(\tau, 0)$ & Initial population of infectious individuals & \\
& of age-since-infection $\tau$ & humans \\
$i(0, t)$ & Newborns at time $t$ & humans year \\
$\Lambda$ & Recruitment rate of susceptible humans & year \\
$m_{0}$ & Natural death rate of susceptible humans & year \\
$m(V)$ & Death rate of infectious humans & mm $^{3}$ virion $^{-1}$ year $^{-1}$ \\
\hline$c_{1}$ & Contact rate between susceptible and infectious humans \\
\hline
\end{tabular}

The total population of infectious individuals from birth to maximal age-since-infection, $A$, is defined as

$$
I(t)=\int_{0}^{A} i(\tau, t) d \tau,
$$

and the total population size of individuals in the population is $N(t)=S(t)+I(t)$. For the sake of introduction to our method, we assume the simplest form for the mortality function [11], $m(V)$, as

$$
m(V(\tau))=m_{0}+\mu_{1} V(\tau)
$$

so that in the absence of the virus, individuals die naturally at rate $m_{0}$. The term $\mu_{1} V(\tau)$ gives the additional host mortality due to the virus. See Coombs et al. [11] for other forms of mortality functions. 


\subsection{Boundedness and Existence of Solutions}

For positive initial data, standard results give that the state variables of the within-host model stay positive for all time, and differential inequalities [14] can be used to establish boundedness of state solutions. The positivity and boundedness of state solutions of the within-host model will be used in the proof of existence of solutions to the between-host system and global stability of disease-free equilibrium of the epidemiological model.

Theorem 2.1. Given the state equations (2.1) - (2.3), with positive initial conditions (2.4), there exist constants $\hat{C}, \tilde{C}, C>0$ such that $0<x(\tau) \leq \hat{C}, 0<y(\tau) \leq \tilde{C}$ and $0<V(\tau) \leq C$, for all $\tau>0$.

We develop a representation formula for the solution (if it exists) to the epidemiological model determined by the methods of integrating factor and characteristics $[7,48]$, and prove the existence and uniqueness of the solution. We use the method of integrating factor to represent the solution of the first-order ordinary differential equation that models the population of susceptible individuals and the method of characteristics for the first-order partial differential equations representing infectious individuals in the population $[31,42]$. A typical approach towards proving well-posedness of a differential equation problem is to write the problem in integral form. To do this, we integrate the differential equation (2.6) along the characteristic line $\tau-t=$ constant and consider cases where $\tau>t$ and $\tau<t$, which gives our representation formula for the solution to the epidemiological model:

$$
\begin{aligned}
S(t)= & S_{0} e^{-\left(m_{0}+\alpha\right) t}+\frac{\Lambda}{m_{0}+\alpha}\left(1-e^{-\left(m_{0}+\alpha\right) t}\right) \\
& +\int_{0}^{t} e^{-\left(m_{0}+\alpha\right)(t-s)} S(s)\left(\alpha-\frac{1}{N(s)} \int_{0}^{A} c_{1} s_{1} V(\tau) i(\tau, s) d \tau\right) d s \\
i(\tau, t)= & \begin{cases}\frac{S(t-\tau)}{N(t-\tau)} e^{-\int_{0}^{\tau} m(V(s)) d s} \int_{0}^{A} c_{1} s_{1} V(r) i(r, t-\tau) d r, & \tau<t \\
i^{0}(\tau-t) e^{-\int_{0}^{t} m(V(\tau-t+s)) d s}, & \tau>t,\end{cases}
\end{aligned}
$$

where $S(t)$ in $(2.9)$ is a representation formula for the solution to the differential equation

$$
\frac{d S}{d t}+\alpha S(t)=\Lambda+\alpha S(t)-\frac{S(t)}{N(t)} \int_{0}^{A} c_{1} s_{1} V(\tau) i(\tau, t) d \tau-m_{0} S(t)
$$

with $\alpha \geq c_{1} s_{1} C>0$. This differential equation is equivalent to equation (2.5).

To prove the existence and uniqueness of solution, we define our state solution space as

$$
\begin{aligned}
X= & \left\{(S, i) \in L^{\infty}(0, T) \times L^{\infty}\left(0, T ; L^{1}(0, A)\right) \mid S(t) \geq \varepsilon>0, \quad i(\tau, t) \geq 0, \sup _{t} S(t)<\infty\right. \\
& \text { and } \left.\sup _{t} \int_{0}^{A} i(\tau, t) d \tau<\infty \text { a.e. } t\right\}
\end{aligned}
$$

where $L^{\infty}(0, A)$ is the space of all essentially bounded functions on $(0, A)$, and $\varepsilon=\min \left\{S_{0}, \frac{\Lambda}{m_{0}+\alpha}\right\}$. We define a map

$$
\mathcal{L}: X \rightarrow X, \quad \mathcal{L}(S, i)=\left(L_{1}(S, i), L_{2}(S, i)\right)
$$

where

$$
\begin{aligned}
L_{1}(S, i)(t)= & S_{0} e^{-\left(m_{0}+\alpha\right) t}+\frac{\Lambda}{m_{0}+\alpha}\left(1-e^{-\left(m_{0}+\alpha\right) t}\right) \\
& +\int_{0}^{t} e^{-\left(m_{0}+\alpha\right)(t-s)} S(s)\left(\alpha-\frac{1}{N(s)} \int_{0}^{A} c_{1} s_{1} V(\tau) i(\tau, s) d \tau\right) d s
\end{aligned}
$$


and

$$
L_{2}(S, i)(\tau, t)= \begin{cases}\frac{S(t-\tau)}{N(t-\tau)} e^{-\int_{0}^{\tau} m(V(s)) d s} \int_{0}^{A} c_{1} s_{1} V(r) i(r, t-\tau) d r, & \tau<t \\ i^{0}(\tau-t) e^{-\int_{0}^{t} m(V(\tau-t+s)) d s}, & \tau>t\end{cases}
$$

The following assumptions will be useful in establishing a Lipschitz property for the within-host and between-host state solutions in terms of control functions (See section 3), and in proving existence and uniqueness of solution to the epidemiological model:

- $S_{0}, m_{0}, \Lambda, c_{1}$ and $s_{1}$ are positive constants,

- $V$ is given, such that $0<V(\tau) \leq C$ for all $\tau>0$

- $m(s)$ is non-negative and Lipschitz continuous,

- $i^{0}(\tau)$ is non-negative for all $\tau \in(0, A)$,

- $\int_{0}^{A} i^{0}(\tau) d \tau \leq M$ and $0<S_{0} \leq M$.

Theorem 2.2. For $T<\infty$, there exists a unique non-negative solution $(S, i)$ to the epidemiological system (2.5)-(2.7).

Proof. First, we show that the map $\mathcal{L}$ maps $X$ into itself. Indeed,

$$
\begin{aligned}
\left|L_{1}(S, i)\right|(t) \leq \mid & S_{0} e^{-\left(m_{0}+\alpha\right) t}+\frac{\Lambda}{m_{0}+\alpha}\left(1-e^{-\left(m_{0}+\alpha\right) t}\right)|+| \alpha \int_{0}^{t} S(s) e^{-\left(m_{0}+\alpha\right)(t-s)} d s \mid \\
+ & \left|\int_{0}^{t} e^{-\left(m_{0}+\alpha\right)(t-s)} \frac{S(s)}{N(s)} \int_{0}^{A} c_{1} s_{1} V(\tau) i(\tau, s) d \tau d s\right| \\
\leq & M+\left|\frac{\Lambda}{m_{0}+\alpha}\left(1-e^{-\left(m_{0}+\alpha\right) T}\right)\right|+\frac{\alpha}{m_{0}+\alpha} \sup _{s} S(s) \\
& +\frac{K_{1}}{m_{0}+\alpha}\left(\sup _{s} \int_{0}^{A} i(\tau, s) d \tau\right)<\infty
\end{aligned}
$$

where $K_{1}$ depends on the contact rate between susceptible and infectious individuals, shedding rate of free virus and the bound on the population of free virus. Next, we estimate the second component.

$$
\begin{aligned}
\int_{0}^{A}\left|L_{2}(S, i)\right|(\tau, t) d \tau= & \int_{0}^{t}\left|\frac{S(t-\tau)}{N(t-\tau)} e^{-\int_{0}^{\tau} m(V(\omega)) d \omega} \int_{0}^{A} c_{1} s_{1} V(r) i(r, t-\tau) d r\right| d \tau \\
& +\int_{t}^{A}\left|i_{1}^{0}(\tau-t) e^{-\int_{0}^{t} m\left(V_{2}(\tau-t+s)\right) d s}\right| d \tau \\
\leq & \int_{0}^{t}\left|\int_{0}^{A} c_{1} s_{1} V(r) i(r, t-\tau) d r\right| d \tau+\int_{0}^{A} i^{0}(\tau) d \tau \\
\leq & K_{2} T\left(\sup _{\xi} \int_{0}^{A} i(\hat{r}, \xi) d \hat{r}\right)+M<\infty
\end{aligned}
$$

where $\hat{r}=r, \xi=t-\tau, K_{2}$ depends on the contact rate between susceptible and infectious individuals, shedding rate of free virus and the bound on the population of free virus. Finally, we show that $L_{1}(S, i)(t) \geq \varepsilon$ and $L_{2}(S, i)(\tau, t) \geq 0$, for all $\tau>0$ and $t>0$. Now, from Theorem 2.1, we obtain

$$
\begin{aligned}
L_{1}(S, i)(t) & \geq S_{0} e^{-\left(m_{0}+\alpha\right) t}+\frac{\Lambda}{m_{0}+\alpha}\left(1-e^{-\left(m_{0}+\alpha\right) t}\right)+\int_{0}^{t} e^{-\left(m_{0}+\alpha\right)(t-s)} S(s)\left(\alpha-c_{1} s_{1} C\right) d s \\
& \geq S_{0} e^{-\left(m_{0}+\alpha\right) t}+\frac{\Lambda}{m_{0}+\alpha}\left(1-e^{-\left(m_{0}+\alpha\right) t}\right) \\
& \geq \varepsilon>0
\end{aligned}
$$


due to the convex combination of $S_{0}$ and $\frac{\Lambda}{m_{0}+\alpha}$. Also, $L_{2}(S, i)(\tau, t) \geq 0$ since $S(t) \geq \varepsilon>0$ and $i(\tau, t) \geq 0$. Hence, $\mathcal{L}$ maps $\mathrm{X}$ to $\mathrm{X}$.

Next, we show that the operator $\mathcal{L}$ admits a unique fixed point. To do this, we define an iterative sequence [42]

$$
\left(S^{(n+1)}(t), i^{(n+1)}(\tau, t)\right)=\left(L_{1}\left(S^{(n)}(t), i^{(n)}(\tau, t)\right), L_{2}\left(S^{(n)}(t), i^{(n)}(\tau, t)\right)\right)
$$

where

$$
\begin{aligned}
S^{(n+1)}(t)= & S_{0} e^{-\left(m_{0}+\alpha\right) t}+\frac{\Lambda}{m_{0}+\alpha}\left(1-e^{-\left(m_{0}+\alpha\right) t}\right) \\
& +\int_{0}^{t} e^{-\left(m_{0}+\alpha\right)(t-s)} S^{(n)}(s)\left(\alpha-\frac{1}{N^{(n)}(s)} \int_{0}^{A} c_{1} s_{1} V(\tau) i^{(n)}(\tau, s) d \tau\right) d s \\
i^{(n+1)}(\tau, t)= & \begin{cases}\frac{S^{(n)}(t-\tau)}{N^{(n)}(t-\tau)} e^{-\int_{0}^{\tau} m(V(s)) d s} \int_{0}^{A} c_{1} s_{1} V(s) i^{(n)}(s, t-\tau) d s, & \tau t \\
i^{0}(\tau-t) e^{-\int_{0}^{t} m(V(\tau-t+s)) d s}, & \tau>t .\end{cases}
\end{aligned}
$$

We set $S^{(0)}(t)=0, i^{(0)}(\tau, t)=0$, and

$$
\begin{aligned}
S^{(1)}(t) & =S_{0} e^{-\left(m_{0}+\alpha\right) t}+\frac{\Lambda}{m_{0}+\alpha}\left(1-e^{-\left(m_{0}+\alpha\right) t}\right) \\
i^{(1)}(\tau, t) & = \begin{cases}0, & \tau<t \\
i^{0}(\tau-t) e^{-\int_{0}^{t} m(V(\tau-t+s)) d s}, & \tau>t,\end{cases}
\end{aligned}
$$

and define a sequence for the total population as

$$
N^{(n)}(t)=S^{(n)}(t)+\int_{0}^{A} i^{(n)}(\tau, t) d \tau
$$

To show that the sequence of functions $\left\{\left(S^{(n)}(t), i^{(n)}(\tau, t)\right)\right\}$ converges for all $n \geq 0$, we introduce the notation

$$
\begin{aligned}
& \mathbb{F}_{n}(t)=\left|S^{(n+1)}(t)-S^{(n)}(t)\right| \\
& \mathbb{I}_{n}(t)=\int_{0}^{A}\left|i^{(n+1)}(\tau, t)-i^{(n)}(\tau, t)\right| d \tau,
\end{aligned}
$$

so that $\mathbb{N}_{n}(t)=\mathbb{F}_{n}(t)+\mathbb{I}_{n}(t)$. Now,

$$
\mathbb{F}_{0}=S_{0} e^{-\left(m_{0}+\alpha\right) t}+\frac{\Lambda}{m_{0}+\alpha}\left(1-e^{-\left(m_{0}+\alpha\right) t}\right) \leq \max \left\{S_{0}, \frac{\Lambda}{m_{0}+\alpha}\right\}
$$

and $\mathbb{I}_{0}=\int_{0}^{A} i^{0}(\tau) d \tau$, so that $\mathbb{N}_{0}=\max \left\{S_{0}, \frac{\Lambda}{m_{0}+\alpha}\right\}+\int_{0}^{A} i^{0}(\tau) d \tau$. Next, for $n=1$, we get

$$
\begin{aligned}
\mathbb{F}_{1} & =\left|S^{(2)}(t)-S^{(1)}(t)\right| \\
& =\left|\int_{0}^{t} e^{-\left(m_{0}+\alpha\right)(t-s)} S^{(1)}(s)\left(\alpha-\frac{1}{N^{(1)}(s)} \int_{0}^{A} c_{1} s_{1} V(\tau) i^{(1)}(\tau, s) d \tau\right) d s\right| \\
& \leq \max \left\{S_{0}, \frac{\Lambda}{m_{0}+\alpha}\right\} \frac{\alpha+c_{1} s_{1} C}{\alpha+m_{0}},
\end{aligned}
$$


and

$$
\begin{aligned}
\mathbb{I}_{1}(t) & =\int_{0}^{A}\left|i^{(2)}(\tau, t)-i^{(1)}(\tau, t)\right| d \tau \\
& =\int_{0}^{t} \frac{S^{(1)}(t-\tau)}{N^{(1)}(t-\tau)} e^{-\int_{0}^{\tau} m(V(s)) d s} \int_{t}^{A} c_{1} s_{1} V(s) i^{0}(s+\tau-t) \frac{\pi(\tau)}{\pi(\tau-s)} d s d \tau \\
& \leq \frac{c_{1} s_{1} C}{m_{0}} \int_{0}^{A} i^{0}(\xi) d \xi
\end{aligned}
$$

where $\xi=s+\tau-t$ and $\pi(\tau)=e^{-\int_{0}^{\tau} m(V(s)) d s}$. Thus, combining equations (2.14) and (2.15), we have $\mathbb{N}_{1}(t) \leq \hat{C} \mathbb{N}_{0}$, for all $t$. Next, we consider the equations for $S$ and $i$, and use induction. First,

$$
\begin{aligned}
\mathbb{F}_{n}(t)= & \left|S^{(n+1)}(t)-S^{(n)}(t)\right| \\
\leq & \alpha \int_{0}^{t} e^{-\left(m_{0}+\alpha\right)(t-\xi)}\left|S^{(n)}(\xi)-S^{(n-1)}(\xi)\right| d \xi \\
& +\int_{0}^{t} e^{-\left(m_{0}+\alpha\right)(t-\xi)}\left|\int_{0}^{A} c_{1} s_{1} V(\tau)\left(\frac{S^{(n)}(\xi) i^{(n)}(\tau, \xi)}{N^{(n)}(\xi)}-\frac{S^{(n-1)}(\xi) i^{(n-1)}(\tau, \xi)}{N^{(n-1)}(\xi)}\right) d \tau\right| d \xi \\
\leq & \alpha \int_{0}^{t}\left|S^{(n)}(\xi)-S^{(n-1)}(\xi)\right| d \xi+\int_{0}^{t} \int_{0}^{A} c_{1} s_{1} V(\tau)|G(\tau, \xi)| d \tau d \xi
\end{aligned}
$$

where

$$
\begin{aligned}
G(\tau, \xi)= & \frac{S^{(n)}(\xi)}{N^{(n)}(\xi)}\left(i^{(n)}(\tau, \xi)-i^{(n-1)}(\tau, \xi)\right)+\frac{i^{(n-1)}(\tau, \xi)}{N^{(n-1)}(\xi)}\left(S^{(n)}(\xi)-S^{(n-1)}(\xi)\right) \\
& +\frac{i^{(n-1)}(\tau, \xi) S^{(n)}(\xi)}{N^{(n)}(\xi)}-\frac{i^{(n-1)}(\tau, \xi) S^{(n)}(\xi)}{N^{(n-1)}(\xi)} \\
= & \frac{S^{(n)}(\xi)}{N^{(n)}(\xi)}\left(i^{(n)}(\tau, \xi)-i^{(n-1)}(\tau, \xi)\right)+\frac{i^{(n-1)}(\tau, \xi)}{N^{(n-1)}(\xi)}\left(S^{(n)}(\xi)-S^{(n-1)}(\xi)\right) \\
& +\frac{i^{(n-1)}(\tau, \xi)}{N^{(n-1)}(\xi)} \frac{S^{(n)}(\xi)}{N^{(n)}(\xi)}\left(S^{(n-1)}(\xi)-S^{(n)}(\xi)\right) \\
& +\frac{i^{(n-1)}(\tau, \xi)}{N^{(n-1)}(\xi)} \frac{S^{(n)}(\xi)}{N^{(n)}(\xi)} \int_{0}^{A}\left(i^{(n-1)}(\sigma, \xi)-i^{(n)}(\sigma, \xi)\right) d \sigma .
\end{aligned}
$$

Since $0<V(\tau) \leq C$, inequality $(2.16)$ gives $\left|S^{(n+1)}(t)-S^{(n)}(t)\right|$

$$
\begin{aligned}
& \leq\left(\alpha+2 c_{1} s_{1} C\right) \int_{0}^{t}\left|S^{(n)}(\xi)-S^{(n-1)}(\xi)\right| d \xi+2 c_{1} s_{1} C \int_{0}^{t} \int_{0}^{A}\left|i^{(n)}(\tau, \xi)-i^{(n-1)}(\tau, \xi)\right| d \tau d \xi \\
& =\left(\alpha+2 c_{1} s_{1} C\right) \int_{0}^{t} \mathbb{F}_{n-1}(\xi) d \xi+2 c_{1} s_{1} C \int_{0}^{t} \mathbb{I}_{n-1}(\xi) d \xi .
\end{aligned}
$$

Thus,

$$
\mathbb{F}_{n}(t) \leq K_{3} \int_{0}^{t}\left(\mathbb{F}_{n-1}(\xi)+\mathbb{I}_{n-1}(\xi)\right) d \xi
$$

where $K_{3}$ depends on the contact rate between susceptible and infectious individuals, shedding rate of free virus and the bound on the population of free virus. Next, we consider the second component. 


$$
\begin{aligned}
\mathbb{I}_{n}(t) & =\int_{0}^{A}\left|i^{(n+1)}(\tau, t)-i^{(n)}(\tau, t)\right| d \tau \\
& \leq \int_{0}^{t} \int_{0}^{A} c_{1} s_{1} V(\sigma)\left|\frac{S^{(n)}(t-\tau) i^{(n)}(\sigma, t-\tau)}{N^{(n)}(t-\tau)}-\frac{S^{(n-1)}(t-\tau) i^{(n-1)}(\sigma, t-\tau)}{N^{(n-1)}(t-\tau)}\right| d \sigma d \tau \\
& \leq K_{4} \int_{0}^{t} \mathbb{F}_{n-1}(\xi) d \xi+K_{4} \int_{0}^{t} \mathbb{I}_{n-1}(\xi) d \xi,
\end{aligned}
$$

where we have mimicked equations (2.16) and (2.18), and used the substitution $\xi=t-\tau$. Since $\mathbb{N}_{n}(t)=$ $\mathbb{F}_{n}(t)+\mathbb{I}_{n}(t)$, combining inequalities $(2.19)$ and $(2.20)$, we see that $\mathbb{N}_{n}(t)$ satisfies the recurrence relation

$$
\mathbb{N}_{n}(t) \leq K \int_{0}^{t} \mathbb{N}_{n-1}(\xi) d \xi, \text { with } \quad \mathbb{N}_{1}(t) \leq \hat{C} \mathbb{N}_{0}
$$

where $K=K_{3}+K_{4}$. Notice that

$$
\mathbb{N}_{2}(t) \leq K \int_{0}^{t} \mathbb{N}_{1}(\xi) d \xi \leq K \hat{C} \mathbb{N}_{0} t
$$

and

$$
\mathbb{N}_{3}(t) \leq K \int_{0}^{t} K \hat{C} \mathbb{N}_{0} \xi d \xi=\hat{C} \mathbb{N}_{0} \frac{K^{2} t^{2}}{2}
$$

Thus, by induction, it follows that

$$
\mathbb{N}_{n}(t) \leq \hat{C} \mathbb{N}_{0} \frac{K^{n-1} t^{n-1}}{(n-1) !} \leq \hat{C} \mathbb{N}_{0} \frac{K^{n-1} T^{n-1}}{(n-1) !} .
$$

Now, the remainder term of the sequence $\left\{S^{(n)}(t)\right\}$ is such that

$$
\left|S^{(n+m)}(t)-S^{(n)}(t)\right| \leq \sum_{j=n+1}^{n+m} \mathbb{N}_{j}(t) \leq \hat{C} \mathbb{N}_{0} \sum_{j=n+1}^{\infty} \frac{K^{j-1} T^{j-1}}{(j-1) !} \rightarrow 0, \quad \text { as } \quad n \rightarrow \infty .
$$

Also, using the notation in (2.13) and the definition of $\mathbb{N}_{n}(t)$, we have

$$
\begin{aligned}
\int_{0}^{A}\left|i^{(n+m)}(\tau, t)-i^{(n)}(\tau, t)\right| d \tau & \leq \sum_{j=n+1}^{n+m} \int_{0}^{A}\left|i^{(j)}(\tau, t)-i^{(j-1)}(\tau, t)\right| d \tau \\
& \leq \sum_{j=n+1}^{n+m} \mathbb{N}_{j}(t) \\
& \leq \hat{C} \mathbb{N}_{0} \sum_{j=n+1}^{\infty} \frac{K^{j-1} T^{j-1}}{(j-1) !} \rightarrow 0 \text { as } n \rightarrow \infty
\end{aligned}
$$

Thus, the sequence $\left\{\left(S^{(n)}(t), i^{(n)}(\tau, t)\right)\right\}$ generated by the iterative process $(2.12)$ is a Cauchy sequence in $X$, and is therefore convergent, since $X$ is complete. Thus, there exists $(S(t), i(\tau, t))$ in $X$ which is the limit of the given sequence. From the iterative process $(2.12)$ and definition of the operator $\mathcal{L}$,

$$
\mathcal{L}(S(t), i(\tau, t))=(S(t), i(\tau, t))
$$

it follows that the limit $(S(t), i(\tau, t))$ is a fixed point of the operator $\mathcal{L}$. This establishes the existence of solution to the epidemiological model for all $T<\infty$. 
We prove uniqueness by assuming the existence of two solutions $(S(t), i(\tau, t))$ and $(\bar{S}(t), \bar{i}(\tau, t))$ for which

$$
(S(t), i(\tau, t))=\left(L_{1}(S(t), i(\tau, t)), L_{2}(S(t), i(\tau, t))\right)
$$

and

$$
(\bar{S}(t), \bar{i}(\tau, t))=\left(L_{1}(\bar{S}(t), \bar{i}(\tau, t)), L_{2}(\bar{S}(t), \bar{i}(\tau, t))\right) .
$$

We substitute $(S(t), i(\tau, t))$ and $(\bar{S}(t), \bar{i}(\tau, t))$ in place of $\left(S^{(n)}(t), i^{n}(\tau, t)\right)$ and $\left(S^{(n-1)}(t), i^{(n-1)}(\tau, t)\right)$, respectively, in the proof of existence of solution above, and set

$$
\hat{\mathbb{F}}(t)=|S(t)-\bar{S}(t)|, \quad \text { and } \quad \hat{\mathbb{I}}(t)=\int_{0}^{A}|i(\tau, t)-\bar{i}(\tau, t)| d \tau .
$$

This gives $\hat{\mathbb{N}}(t) \leq K \int_{0}^{t} \hat{\mathbb{N}}(\xi) d \xi$, so that by Gronwall's inequality in integral form, $\hat{\mathbb{N}}(t) \equiv 0$. Thus, $\hat{\mathbb{F}}(t)+\hat{\mathbb{I}}(t)=0, \quad \forall t>0$. Since $\hat{\mathbb{F}}(t) \geq 0$, and $\hat{\mathbb{I}}(t) \geq 0$, with $\hat{\mathbb{F}}(t)+\hat{\mathbb{I}}(t)=0$, it follows that $\hat{\mathbb{F}}(t)=\hat{\mathbb{I}}(t)=0$, for all $t>0$. Hence, the solution, $(S(t), i(\tau, t))$, to the epidemiological model is unique.

\subsection{Equilibria and Stability Analysis of the Epidemiological Model}

In this subsection, we derive an explicit expression for the basic reproduction number of the epidemiological model, calculate steady state solutions and study stability of equilibrium points.

\subsubsection{Basic Reproduction Number and Equilibria}

For age-structured models, we use the notions of survival functions or probabilities in the computation of the basic reproduction number, $\mathcal{R}_{0}$. Now, let $\mathcal{F}(\tau)$ be the probability that a newly infected individual remains infected until time-since-infection $\tau$, and $\widehat{\beta}(\tau)$ denote the average number of newly infected individuals that an infectious individual will produce per unit time when infected for a total time $\tau$, then the basic reproduction number is given by [28]

$$
\mathcal{R}_{0}=\int_{0}^{A} \widehat{\beta}(\tau) \mathcal{F}(\tau) d \tau
$$

In order to derive an explicit expression for the basic reproduction number, $\mathcal{R}_{0}$, of the age-structured epidemiological model, we compute the disease-free equilibrium, linearize the system around the disease-free equilibrium and determine conditions for its stability. Now, the disease-free equilibrium is $\left(S^{*}, i^{*}(\tau)\right)=$ $\left(\frac{\Lambda}{m_{0}}, 0\right)$. We consider solutions nearby $\left(S^{*}, i^{*}(\tau)\right)$ by setting

$$
x(t)=S(t)-S^{*} \quad \text { and } \quad i(\tau, t)=z(\tau, t) .
$$

Substituting the perturbed solutions into equations (2.5)-(2.7), we have the following linearized system

$$
\begin{aligned}
\frac{d x}{d t} & =-\int_{0}^{A} c_{1} s_{1} V(\tau) z(\tau, t) d \tau-m_{0} x(t) \\
\frac{\partial z(\tau, t)}{\partial t}+\frac{\partial z(\tau, t)}{\partial \tau} & =-m(V(\tau)) z(\tau, t) \\
z(0, t) & =\int_{0}^{A} c_{1} s_{1} V(\tau) z(\tau, t) d \tau .
\end{aligned}
$$

We seek a solution to equation (2.22) of the form

$$
z(\tau, t)=\bar{z}(\tau) e^{\lambda t}
$$


where $\lambda$ is either a real or complex number. Substituting this solution into equations (2.22)-(2.23), we have the following eigenvalue problem

$$
\begin{aligned}
\frac{d \bar{z}(\tau)}{d \tau} & =-(\lambda+m(V(\tau))) \bar{z}(\tau) \\
\bar{z}(0) & =\int_{0}^{A} c_{1} s_{1} V(\tau) \bar{z}(\tau) d \tau .
\end{aligned}
$$

The explicit solution to the differential equation gives

$$
\bar{z}(0)=\int_{0}^{A} c_{1} s_{1} V(\tau) \bar{z}(0) e^{-\lambda \tau} e^{-\int_{0}^{\tau} m(V(s)) d s} d \tau
$$

Dividing both sides of equation (2.26) by $z(0)$, we obtain the characteristic equation $G(\lambda)=1$, where

$$
G(\lambda)=\int_{0}^{A} c_{1} s_{1} V(\tau) e^{-\lambda \tau} e^{-\int_{0}^{\tau} m(V(s)) d s} d \tau .
$$

This characteristic equation will be used to study stability of the disease-free equilibrium. Now, we define the basic reproduction number, $\mathcal{R}_{0}$, of the epidemiological model as $\mathcal{R}_{0}=G(0)[10,40,45$, 46], so that

$$
\mathcal{R}_{0}=\int_{0}^{A} c_{1} s_{1} V(\tau) e^{-\int_{0}^{\tau} m(V(s)) d s} d \tau
$$

where the quantity $\pi(\tau)=e^{-\int_{0}^{\tau} m(V(s)) d s}$ is the probability of survival in the infected class from onset of infection to age-since-infection $\tau$.

Theorem 2.3. The epidemiological model has a unique endemic equilibrium, $\left(S^{*}, i^{*}(\tau)\right)$, if $\mathcal{R}_{0}>1$.

Proof. The equilibria of the epidemiological model are obtained by setting the time derivatives of the model to zero:

$$
\begin{aligned}
0 & =\Lambda-\frac{S}{N} \int_{0}^{A} c_{1} s_{1} V(\tau) i(\tau) d \tau-m_{0} S \\
\frac{d i(\tau)}{d \tau} & =-m(V(\tau)) i(\tau) \\
i(0) & =\frac{S}{N} \int_{0}^{A} c_{1} s_{1} V(\tau) i(\tau) d \tau .
\end{aligned}
$$

The endemic equilibrium is obtained as follows. First, we solve the differential equation (2.30) to have

$$
i^{*}(\tau)=i^{*}(0) e^{-\int_{0}^{\tau} m(V(s)) d s} .
$$

Next, we substitute the expression for $i^{*}$ into equation (2.29):

$$
0=\Lambda-\frac{S^{*}}{N} \int_{0}^{A} c_{1} s_{1} V(\tau) i^{*}(0) e^{-\int_{0}^{\tau} m(V(s)) d s} d \tau-m_{0} S^{*}
$$

Thus, from equations (2.31), (2.32) and (2.33), we obtain $i^{*}(0)$ as follows:

$$
\begin{aligned}
i^{*}(0) & =\frac{S^{*}}{N} \int_{0}^{A} c_{1} s_{1} V(\tau) i^{*}(0) e^{-\int_{0}^{\tau} m(V(s)) d s} d \tau \\
& =\Lambda-m_{0} S^{*}
\end{aligned}
$$


From equations (2.31) and (2.32), and the total population at equilibrium $N^{*}=S^{*}+\int_{0}^{A} i^{*}(\tau) d \tau$, we obtain

$$
\frac{S^{*}}{N^{*}}=\frac{1}{\mathcal{R}_{0}} \quad \text { and } \quad \frac{i^{*}(0)}{N^{*}}=\frac{\mathcal{R}_{0}-1}{\xi \mathcal{R}_{0}},
$$

where $\xi=\int_{0}^{A} e^{-\int_{0}^{\tau} m(V(s)) d s} d \tau$. Also, from equations (2.34) and (2.35), we obtain $N^{*}=\frac{\Lambda \xi \mathcal{R}_{0}}{\mathcal{R}_{0}-1+m_{0} \xi}$. Finally, from equations $(2.32)$ and (2.35), we obtain the endemic equilibrium point $\left(S^{*}, i^{*}(\tau)\right)$, where

$$
\left(S^{*}, i^{*}(\tau)\right)=\left(\frac{\Lambda \int_{0}^{A} e^{-\int_{0}^{\tau} m(V(s)) d s} d \tau}{\mathcal{R}_{0}-1+m_{0} \int_{0}^{A} e^{-\int_{0}^{\tau} m(V(s)) d s} d \tau}, \frac{\Lambda\left(\mathcal{R}_{0}-1\right) e^{-\int_{0}^{\tau} m(V(s)) d s}}{\mathcal{R}_{0}-1+m_{0} \int_{0}^{A} e^{-\int_{0}^{\tau} m(V(s)) d s} d \tau}\right),
$$

which is biologically feasible if $\mathcal{R}_{0}>1$.

\subsubsection{Stability Analysis}

To study the local stability of equilibria, we linearize the model around each of the equilibrium points, and consider an exponential solution to the linearized system.

Theorem 2.4. The disease-free equilibrium is locally asymptotically stable if $\mathcal{R}_{0}<1$ and unstable if $\mathcal{R}_{0}>1$.

Proof. If $\lambda \in \Re$, then from equation (2.27),

$$
G^{\prime}(\lambda)=-\int_{0}^{A} c_{1} s_{1} V(\tau) \tau e^{-\lambda \tau} e^{-\int_{0}^{\tau} m(V(s)) d s} d \tau<0,
$$

since $V$ is nonnegative and bounded. Thus, $G$ is a decreasing function of $\lambda$, with $\lim _{\lambda \rightarrow \infty} G(\lambda)=0$. Therefore, when $\mathcal{R}_{0}=G(0)>1$, there exists a unique positive real solution to the equation $G(\lambda)=1$. Hence, the disease-free equilibrium is unstable when $\mathcal{R}_{0}>1[40,45,46]$.

On the other hand, $\lim _{\lambda \rightarrow-\infty} G(\lambda)=+\infty$. Thus, when $\mathcal{R}_{0}=G(0)<1$, there exists a unique real and negative solution to the equation $G(\lambda)=1$. Next, we assume that $\lambda$ is complex and let $\lambda=\xi+i \eta$ be an arbitrary complex solution to the characteristic equation $G(\lambda)=1$. Then

$$
\begin{aligned}
1 & =|G(\xi+i \eta)| \\
& \leq \int_{0}^{A} c_{1} s_{1} V(\tau) e^{-\xi \tau}\left|e^{-i \eta \tau}\right| e^{-\int_{0}^{\tau} m(V(s)) d s} d \tau \\
& =\int_{0}^{A} c_{1} s_{1} V(\tau) e^{-\xi \tau} e^{-\int_{0}^{\tau} m(V(s)) d s} d \tau=: G(\operatorname{Re}(\lambda)) .
\end{aligned}
$$

If $\operatorname{Re}(\lambda) \geq 0$, then

$$
1=|G(\lambda)| \leq G(\operatorname{Re}(\lambda)) \leq G(0)=\mathcal{R}_{0}<1,
$$

which is absurd. Thus, all roots of the equation $G(\lambda)=1$ are either real and negative or complex with negative real parts when $\mathcal{R}_{0}<1$. Hence the disease-free equilibrium is locally asymptotically stable when $\mathcal{R}_{0}<1$.

Theorem 2.5. The disease-free equilibrium is globally stable if $\mathcal{R}_{0}<1$.

Proof. The general approach in showing global stability of the disease-free equilibrium is to view the boundary condition as a function of time, solve the PDE along characteristic lines and substitute the solution into the expression for the boundary condition to obtain an integral equation. Now, let

$$
g(t)=\frac{S(t)}{N(t)} K(t),
$$


where

$$
K(t)=\int_{0}^{A} c_{1} s_{1} V(\tau) i(\tau, t) d \tau
$$

We derive an integral equation for $K(t)$ by using the following solution to the partial differential equation (2.6):

$$
i(\tau, t)= \begin{cases}\frac{S(t-\tau)}{N(t-\tau)} e^{-\int_{0}^{\tau} m(V(s)) d s} \int_{0}^{A} c_{1} s_{1} V(r) i(r, t-\tau) d r, & \tau<t \\ i^{0}(\tau-t) e^{-\int_{0}^{t} m(V(\tau-t+s)) d s}, & \tau>t .\end{cases}
$$

Substituting the expression for $i(\tau, t)$ in $K(t)$, we obtain

$$
\begin{aligned}
K(t)= & \int_{0}^{t} c_{1} s_{1} K(t-\tau) V(\tau) \frac{S(t-\tau)}{N(t-\tau)} e^{-\int_{0}^{\tau} m(V(s)) d s} d \tau \\
& +\int_{t}^{A} c_{1} s_{1} V(\tau) i^{0}(\tau-t) e^{-\int_{0}^{t} m(V(\tau-t+s)) d s} d \tau \\
\leq & \int_{0}^{t} c_{1} s_{1} K(t-\tau) V(\tau) e^{-\int_{0}^{\tau} m(V(s)) d s} d \tau+\int_{t}^{A} c_{1} s_{1} V(\tau) i^{0}(\tau-t) d \tau
\end{aligned}
$$

Since for all $\tau \in(0, A), 0<V(\tau) \leq C$, it follows from the definition of (2.36) that

$$
\limsup _{t} K(t) \leq c_{1} s_{1} C \limsup _{t} \int_{0}^{A} i(\tau, t) d \tau<\infty .
$$

Thus, taking the limsup of both sides of equation (2.37) as $t \rightarrow \infty$, we have

$$
\limsup _{t \rightarrow \infty} K(t) \leq \mathcal{R}_{0} \limsup _{t \rightarrow \infty} K(t)
$$

which holds only if $\limsup _{t \rightarrow \infty} K(t)=0$. This gives $\lim \sup _{t \rightarrow \infty} i(\tau, t)=0$ for every fixed $\tau$. The solution to the equation that models susceptible individuals in the population is

$$
\begin{aligned}
S(t)= & -\int_{0}^{t} e^{-m_{0}(t-s)} \frac{S(s)}{N(s)} \int_{0}^{A} c_{1} s_{1} V(\tau) i(\tau, s) d \tau d s+S_{0} e^{-m_{0} t} \\
& +\frac{\Lambda}{m_{0}}\left(1-e^{-m_{0} t}\right) \rightarrow \frac{\Lambda}{m_{0}} \quad \text { as } \quad t \rightarrow \infty .
\end{aligned}
$$

Hence the disease-free equilibrium is globally stable when $\mathcal{R}_{0}<1$.

Theorem 2.6. The endemic equilibrium $\left(S^{*}, i^{*}(\tau)\right)$ is locally asymptotically stable if $\mathcal{R}_{0}>1$ and the maximal age of infection, $A$, is sufficiently large.

Proof. We consider solutions near the endemic equilibrium by setting

$$
x(t)=S(t)-S^{*}, \quad z(\tau, t)=i(\tau, t)-i^{*}(\tau)
$$

so that the total population is $N(t)=N^{*}+n(t)$. Substituting the perturbed solutions into equations (2.5)-(2.7), we have the following linearized system: 


$$
\begin{aligned}
\frac{d x}{d t}= & -\frac{x}{N^{*}} \int_{0}^{A} c_{1} s_{1} V(\tau) i^{*}(\tau) d \tau+\frac{S^{*}}{N^{*}} \frac{n}{N^{*}} \int_{0}^{A} c_{1} s_{1} V(\tau) i^{*}(\tau) d \tau \\
& -\frac{S^{*}}{N^{*}} \int_{0}^{A} c_{1} s_{1} V(\tau) z(\tau, t) d \tau-m_{0} x \\
\frac{\partial z(\tau, t)}{\partial t}+\frac{\partial z(\tau, t)}{\partial t}= & -m(V(\tau)) z(\tau, t) \\
z(0, t)= & \frac{x}{N^{*}} \int_{0}^{A} c_{1} s_{1} V(\tau) i^{*}(\tau) d \tau-\frac{S^{*}}{N^{*}} \frac{n}{N^{*}} \int_{0}^{A} c_{1} s_{1} V(\tau) i^{*}(\tau) d \tau \\
& +\frac{S^{*}}{N^{*}} \int_{0}^{A} c_{1} s_{1} V(\tau) z(\tau, t) d \tau .
\end{aligned}
$$

We seek for solutions to equations (2.38)-(2.40) of the form

$$
x(t)=\bar{x} e^{\lambda t} \quad \text { and } \quad z(\tau, t)=\bar{z}(\tau) e^{\lambda t},
$$

where $\bar{x}$ and $\bar{z}(\tau)$ are to be determined. This gives

$$
\begin{aligned}
\lambda \bar{x}= & -\frac{\bar{x}}{N^{*}} \int_{0}^{A} c_{1} s_{1} V(\tau) i^{*}(\tau) d \tau+\frac{S^{*}}{N^{*}} \frac{\bar{n}}{N^{*}} \int_{0}^{A} c_{1} s_{1} V(\tau) i^{*}(\tau) d \tau \\
& -\frac{S^{*}}{N^{*}} \int_{0}^{A} c_{1} s_{1} V(\tau) \bar{z}(\tau) d \tau-m_{0} \bar{x} \\
\frac{d \bar{z}(\tau)}{d \tau}= & -(\lambda+m(V(\tau))) \bar{z}(\tau) \\
\bar{z}(0)= & \frac{\bar{x}}{N^{*}} \int_{0}^{A} c_{1} s_{1} V(\tau) i^{*}(\tau) d \tau-\frac{S^{*}}{N^{*}} \frac{\bar{n}}{N^{*}} \int_{0}^{A} c_{1} s_{1} V(\tau) i^{*}(\tau) d \tau \\
& +\frac{S^{*}}{N^{*}} \int_{0}^{A} c_{1} s_{1} V(\tau) \bar{z}(\tau) d \tau
\end{aligned}
$$

where $\bar{n}=\bar{x}+\int_{0}^{A} \bar{z}(\tau) d \tau$. Solving the differential equation (2.42), we obtain

$$
\bar{z}(\tau)=\bar{z}(0) e^{-\lambda \tau} e^{-\int_{0}^{\tau} m(V(s)) d s} .
$$

From equations (2.41) and (2.43),

$$
\bar{z}(0)=-\left(\lambda+m_{0}\right) \bar{x} .
$$

Using the definitions of $\bar{n}, \bar{z}(\tau)$ and $\bar{z}(0)$, and setting $\tilde{\alpha}=\int_{0}^{A} c_{1} s_{1} V(\tau) i^{*}(\tau) d \tau$ in equation (2.41), we obtain the characteristic equation

$$
1=\frac{\tilde{\alpha}}{N^{*}\left(\lambda+m_{0}\right)}\left(\frac{S^{*}}{N^{*}}-1\right)+\frac{S^{*}}{N^{*}} \int_{0}^{A} c_{1} s_{1} V(\tau) e^{-\lambda \tau} \pi(\tau) d \tau-\frac{\tilde{\alpha}}{N^{*}} \frac{S^{*}}{N^{*}} \int_{0}^{A} e^{-\lambda \tau} \pi(\tau) d \tau .
$$

Using $m(V(\tau))=m_{0}+\mu_{1} V(\tau)$ and integration by parts, we obtain $\int_{0}^{A} c_{1} s_{1} V(\tau) e^{-\lambda \tau} \pi(\tau) d \tau$

$$
\begin{aligned}
& =\frac{c_{1} s_{1}}{\mu_{1}} \int_{0}^{A} \mu_{1} V(\tau) e^{-\lambda \tau} e^{-m_{0} \tau} e^{-\mu_{1} \int_{0}^{\tau} V(s) d s} d \tau \\
& =\frac{c_{1} s_{1}}{\mu_{1}}\left(1-e^{-\left(\lambda+m_{0}\right) A} e^{-\mu_{1} \int_{0}^{A} V(s) d s}\right)-\frac{c_{1} s_{1}}{\mu_{1}}\left(\lambda+m_{0}\right) \int_{0}^{A} e^{-\lambda \tau} \pi(\tau) d \tau .
\end{aligned}
$$


Thus,

$$
\mu_{1} \int_{0}^{A} V(\tau) e^{-\lambda \tau} \pi(\tau) d \tau+\left(\lambda+m_{0}\right) \int_{0}^{A} e^{-\lambda \tau} \pi(\tau) d \tau=1-e^{-\left(\lambda+m_{0}\right) A} e^{-\mu_{1} \int_{0}^{A} V(s) d s} .
$$

From equation (2.46), the characteristic equation (2.45) becomes

$1+\frac{\tilde{\alpha}}{N^{*}\left(\lambda+m_{0}\right)}$

$$
=\frac{1}{\mathcal{R}_{0}}\left(\frac{\tilde{\alpha}}{N^{*}\left(\lambda+m_{0}\right)} \frac{\mu_{1}}{c_{1} s_{1}}+1\right) \int_{0}^{A} c_{1} s_{1} V(\tau) e^{-\lambda \tau} \pi(\tau) d \tau+\frac{1}{\mathcal{R}_{0}} \frac{\tilde{\alpha}}{N^{*}\left(\lambda+m_{0}\right)} e^{-\lambda A} \pi(A),
$$

so that

$$
\mathcal{L}(\lambda)=\frac{\lambda+m_{0}+\frac{\tilde{\alpha}}{N^{*}}}{\lambda+m_{0}+\frac{\tilde{\alpha}}{N^{*}} \frac{\mu_{1}}{c_{1} s_{1}}}
$$

where

$$
\mathcal{L}(\lambda)=\frac{1}{\mathcal{R}_{0}} \int_{0}^{A} c_{1} s_{1} V(\tau) e^{-\lambda \tau} \pi(\tau) d \tau+\frac{\frac{1}{\mathcal{R}_{0}} \frac{\tilde{\alpha}}{N^{*}\left(\lambda+m_{0}\right)}}{\frac{\tilde{\alpha}}{N^{*}\left(\lambda+m_{0}\right)} \frac{\mu_{1}}{c_{1} s_{1}}+1} e^{-\lambda A} \pi(A) .
$$

When $\lambda=0$ in equation (2.46), we obtain

$$
\mu_{1} \int_{0}^{A} V(\tau) \pi(\tau) d \tau=1-\pi(A)-m_{0} \int_{0}^{A} \pi(\tau) d \tau
$$

so that $\mu_{1} \int_{0}^{A} V(\tau) \pi(\tau) d \tau<1$. Since $\mathcal{R}_{0}>1$, it follows that $\frac{c_{1} s_{1}}{\mu_{1}}>1$. Now, let $\lambda=a+i b$ be an arbitrary complex solution (if it exists) of the characteristic equation $(2.47)$. If $\Re(\lambda)>0$, then

$$
\left|\frac{\lambda+m_{0}+\frac{\tilde{\alpha}}{N^{*}}}{\lambda+m_{0}+\frac{\tilde{\alpha}}{N^{*}} \frac{\mu_{1}}{c_{1} s_{1}}}\right|>1 \text { and } \quad|\mathcal{L}(\lambda)|<1
$$

if, and only if, $A$ is sufficiently large. Thus, the case $\Re(\lambda)>0$ gives a contradiction. If $\Re(\lambda)=0(a=0)$, we rewrite the characteristic equation $(2.47)$ as

$$
i b+m_{0}+\frac{\tilde{\alpha}}{N^{*}}=\frac{1}{\mathcal{R}_{0}}\left(\frac{\tilde{\alpha}}{N^{*}} \frac{\mu_{1}}{c_{1} s_{1}}+m_{0}+i b\right) \int_{0}^{A} c_{1} s_{1} V(\tau) e^{-i b \tau} \pi(\tau) d \tau+\frac{1}{\mathcal{R}_{0}} \frac{\tilde{\alpha}}{N^{*}} e^{-i b A} \pi(A) .
$$

Equating imaginary parts of equation (2.48), we obtain

$$
\begin{aligned}
b\left(\mathcal{R}_{0}-\int_{0}^{A} c_{1} s_{1} V(\tau) \cos (b \tau) \pi(\tau) d \tau\right)= & -\left(\frac{\tilde{\alpha}}{N^{*}} \frac{\mu_{1}}{c_{1} s_{1}}+m_{0}\right) \int_{0}^{A} c_{1} s_{1} V(\tau) \sin (b \tau) \pi(\tau) d \tau \\
& -\frac{\tilde{\alpha}}{N^{*}} \sin (b A) \pi(A)
\end{aligned}
$$

Now, using the expression for the basic reproduction number (2.28),

$$
\begin{aligned}
\left.\mathcal{R}_{0}-\int_{0}^{A} c_{1} s_{1} V(\tau) \cos (b \tau) \pi(\tau) d \tau\right) & \left.=2 \int_{0}^{A} c_{1} s_{1} V(\tau) \sin ^{2}\left(\frac{b \tau}{2}\right) \pi(\tau) d \tau\right) \\
& >2 c_{1} s_{1} \varepsilon^{\prime} \pi\left(\alpha_{2}\right) \int_{\alpha_{1}}^{\alpha_{2}} \sin ^{2}\left(\frac{b \tau}{2}\right) d \tau \\
& =\tilde{K}_{1} \pi\left(\alpha_{2}\right)>0, \text { for }\left(\alpha_{1}, \alpha_{2}\right) \subset[0, A],
\end{aligned}
$$


where $\varepsilon^{\prime}$ is a lower bound on $V(\tau)$ for $\tau \in[0, A]$. Now, choose $B^{*}$ such that

$$
B^{*} \tilde{K}_{1} \pi\left(\alpha_{2}\right)>\left(\frac{\tilde{\alpha}}{N^{*}} \frac{\mu_{1}}{c_{1} s_{1}}+m_{0}\right) \int_{0}^{A} c_{1} s_{1} V(\tau) \pi(\tau) d \tau+\frac{\tilde{\alpha}}{N^{*}} \pi(A),
$$

then for $b>B^{*}$, equation (2.49) is untenable. For $b<B^{*}$, the right-hand side of equation (2.47) gives

$$
\left|\frac{m_{0}+\frac{\tilde{\alpha}}{N^{*}}+i b}{\frac{\tilde{\alpha}}{N^{*}} \frac{\mu_{1}}{c_{1} s_{1}}+m_{0}+i b}\right|>\frac{\sqrt{\left(m_{0}+\frac{\tilde{\alpha}}{N^{*}}\right)^{2}+B^{* 2}}}{\sqrt{\left(\frac{\tilde{\alpha}}{N^{*}} \frac{\mu_{1}}{c_{1} s_{1}}+m_{0}\right)^{2}+B^{* 2}}}>1,
$$

and the left-hand side of equation (2.47) gives

$$
|\mathcal{L}(\lambda)| \leq 1+\frac{1}{\mathcal{R}_{0}} \frac{\tilde{\alpha}}{N^{*}}\left(\frac{e^{-m_{0} A}}{\frac{\tilde{\alpha}}{N^{*}} \frac{\mu_{1}}{c_{1} s_{1}}+m_{0}}\right)<\frac{\sqrt{\left(m_{0}+\frac{\tilde{\alpha}}{N^{*}}\right)^{2}+B^{* 2}}}{\sqrt{\left(\frac{\tilde{\alpha}}{N^{*}} \frac{\mu_{1}}{c_{1} s_{1}}+m_{0}\right)^{2}+B^{* 2}}}
$$

if $A$ is sufficiently large. Also, the case $\Re(\lambda)=0$ gives a contradiction. Thus, all solutions of the characteristic equation $(2.47)$ have negative real parts. Hence, the endemic equilibrium, $\left(S^{*}, i^{*}(\tau)\right)$, is locally asymptotically stable when $\mathcal{R}_{0}>1$.

\section{Optimal Control Problem}

Optimal control theory can be used to design intervention strategies for the control of infectious diseases and has been applied in decoupled immunological and epidemiological models of HIV [9, 18, 32-34]. In this section, we apply optimal control theory in a coupled within-host and between-host model of HIV with age (age-since-infection) structure.

The theory of age-structured models abound in the literature [3,48]. In 1974, Gurtin and MacCamy [25] introduced the first model of nonlinear continuous age-dependent population dynamics.

Optimal control of first-order PDEs coming from age-structured models requires more analysis for justification than optimal control of parabolic PDE or differential equations. There has been only a small amount of work on specific applications of optimal control to age-structure equations. Brokate [8] developed maximum principles for an optimal harvesting problem and a problem of optimal birth control. Barbu and Iannelli [6,7] considered and optimal control problem for a Gurtin-MacCamy [48] type system, describing the evolution of an age-structured population. Anita [3,4] investigated an optimal control problem for a nonlinear age-dependent population dynamics. Murphy and Smith [43] studied the optimal harvesting of an age-structured population, where the McKendrick model of population dynamics was used. These authors considered age-structured population models for a single population. Fister and Lenhart [20], on the other hand, considered optimal harvesting control for a competitive age-structured model, comprising two first-order partial differential equations. Also, Fister and Lenhart [19] investigated an optimal harvesting control in a predator-prey model in which the prey population is represented by a first-order partial differential equation with age-structure and the predator is represented by an ordinary differential equation in time. A key tool for the existence and uniqueness of optimal solution is Ekeland's variational principle [13].

In our coupled model, we incorporate two controls which aim at curtailing the transmission rate and virion production. Thus, our within-host model with control is:

$$
\begin{aligned}
& \frac{d x}{d \tau}=r-\beta_{1}\left(1-u_{1}(\tau)\right) V(\tau) x(\tau)-\mu x(\tau) \\
& \frac{d y}{d \tau}=\beta_{1}\left(1-u_{1}(\tau)\right) V(\tau) x(\tau)-d_{1} y(\tau) \\
& \frac{d V}{d \tau}=\nu_{1}\left(1-u_{2}(\tau)\right) d_{1} y(\tau)-\left(\delta_{1}+s_{1}\right) V(\tau)-\hat{\beta}_{1}\left(1-u_{1}(\tau)\right) V(\tau) x(\tau)
\end{aligned}
$$


where the parameters are as defined in Table 1 . The control functions $u_{1}$ and $u_{2}$ are bounded Lebesgue integrable functions and represent the transmission and viral production suppressing drugs, respectively. The coefficient, $1-u_{1}(t)$, represents the drug effect that reduces transmission of healthy cells to infected cells as a result of interaction with the virus, while the coefficient $1-u_{2}(t)$ gives the effect of another drug that reduces the production of virions. The upper bounds on the controls give the efficacy of the transmission and virion production suppressing drugs. If $u_{1}=0$ and $u_{2}=0$ there is no inhibition of transmission and virion production.

\subsection{Sensitivity and Adjoint Systems}

Below, we formulate an objective functional for our coupled system, with the goal of minimizing free virus and infected individuals:

$$
\begin{aligned}
J\left(u_{1}, u_{2}\right) & =\int_{0}^{T} \int_{0}^{A} A_{1} i(\tau, t) V(\tau) d \tau d t \\
& +\int_{0}^{T} \int_{0}^{A} i(\tau, t)\left(A_{2} u_{1}(\tau)+A_{3} u_{2}(\tau)\right) d \tau d t+\int_{0}^{A} B\left(u_{1}(\tau)^{2}+u_{2}(\tau)^{2}\right) d \tau,
\end{aligned}
$$

where $A_{1}, A_{2}, A_{3}$ and $B$ are positive constants that balance the relative importance for the terms in $J$. In our objective functional, the first term with $A_{1}$ represents the total of the infected individuals over time and the other two terms represents costs of implementing the controls. The optimal control formulation with equations (3.1)-(3.3), (2.4) and (2.5)-(2.8) is

$$
\min _{\left(u_{1}, u_{2}\right) \in \mathcal{U}} J\left(u_{1}, u_{2}\right)
$$

where the control set $\mathcal{U}$ is

$$
\mathcal{U}=\left\{\left(u_{1}, u_{2}\right) \in\left(L^{\infty}(0, A)\right)^{2} \mid u_{1}:(0, A) \rightarrow\left[0, \tilde{u}_{1}\right], u_{2}:(0, A) \rightarrow\left[0, \tilde{u}_{2}\right]\right\} .
$$

Below, we state without proof, the Ekeland's Variational Principle.

Theorem 3.1. (Ekeland's Variational Principle [13]) Let $(X, d)$ be a complete metric space and $f: X \rightarrow(-\infty, \infty]$ be a lower semicontinuous function, bounded from below and not identically $+\infty$. Let $\varepsilon>0$ and $u \in X$ be such that $f(u) \leq \inf \{f(x) \mid x \in X\}+\varepsilon$. Then for any $\lambda>0$, there exists $u_{\varepsilon} \in X$ such that

(i) $f\left(u_{\varepsilon}\right) \leq f(u)$

(ii) $d\left(u, u_{\varepsilon}\right) \leq \lambda$

(iii) $f\left(u_{\varepsilon}\right)<f(x)+\varepsilon \lambda^{-1} d\left(u_{\varepsilon}, x\right), \quad \forall x \in X \backslash\left\{u_{\varepsilon}\right\}$.

In addition, if $X$ is a Banach space and $f: X \rightarrow(-\infty, \infty]$ is Gâteaux differentiable, then Ekeland's variational principle guarantees the existence of a minimizing sequence for function $f$.

We formulate a Lipschitz property for state variables in our model in terms of the control functions $u_{1}$ and $u_{2}$. This property will be used to prove the existence of sensitivities and optimal control, and the uniqueness of optimal control.

Theorem 3.2. The map $\left(u_{1}, u_{2}\right) \rightarrow(x, y, V, S, i)=(x, y, V, S, i)\left(u_{1}, u_{2}\right)$ is Lipschitz in the following ways:

$$
\begin{aligned}
\text { (i) } \quad & \int_{0}^{A}(|x-\bar{x}|+|y-\bar{y}|+|V-\bar{V}|) d \tau+\int_{0}^{T}|S-\bar{S}| d t+\int_{Q}|i-\bar{i}| d \tau d t \\
\leq & C_{A, T} \int_{0}^{A}\left(\left|u_{1}-\bar{u}_{1}\right|+\left|u_{2}-\bar{u}_{2}\right|\right) d \tau \\
(i i) \quad & \|x-\bar{x}\|_{L^{\infty}(\Omega)}+\|y-\bar{y}\|_{L^{\infty}(\Omega)}+\|V-\bar{V}\|_{L^{\infty}(\Omega)}+\|S-\bar{S}\|_{L^{\infty}(0, T)} \\
& +\|i-\bar{i}\|_{L^{\infty}\left(0, T ; L^{1}(0, A)\right)} \leq \hat{C}_{A, T}\left(\left\|u_{1}-\bar{u}_{1}\right\|_{L^{\infty}(\Omega)}+\left\|u_{2}-\bar{u}_{2}\right\|_{L^{\infty}(\Omega)}\right),
\end{aligned}
$$

where $\Omega=(0, A)$ and $Q=\Omega \times(0, T)$. 
Proof. (i) First, considering equation (3.1), we have

$$
\frac{d}{d \tau}(x-\bar{x})=-\beta_{1}\left(\bar{u}_{1}-u_{1}\right) V x-\beta_{1}\left(1-\bar{u}_{1}\right)(x(V-\bar{V})+\bar{V}(x-\bar{x}))-\mu(x-\bar{x}) .
$$

Integrating from 0 to $\tau$, noting that $x$ and $\bar{x}$ agree at $\tau=0$, we have

$$
|x-\bar{x}|(\tau) \leq C_{1} \int_{0}^{A}\left|u_{1}-\bar{u}_{1}\right| d s+C_{2} \int_{0}^{\tau}(|x-\bar{x}|+|y-\bar{y}|+|V-\bar{V}|) d s,
$$

since $x$ and $V$ are bounded (See Theorem 2.1). Secondly, considering equations (3.2) and (3.3), we have

$$
\begin{aligned}
& |y-\bar{y}|(\tau) \leq C_{1} \int_{0}^{A}\left|u_{1}-\bar{u}_{1}\right| d s+C_{3} \int_{0}^{\tau}(|x-\bar{x}|+|y-\bar{y}|+|V-\bar{V}|) d s \\
& |V-\bar{V}|(\tau) \leq C_{4} \int_{0}^{A}\left(\left|u_{1}-\bar{u}_{1}\right|+\left|u_{2}-\bar{u}_{2}\right|\right) d s+C_{5} \int_{0}^{\tau}(|x-\bar{x}|+|y-\bar{y}|+|V-\bar{V}|) d s .
\end{aligned}
$$

Combining equations (3.5), (3.6) and (3.7), we have

$$
\begin{aligned}
(|x-\bar{x}|+|y-\bar{y}|+|V-\bar{V}|)(\tau) \leq & C_{6} \int_{0}^{A}\left(\left|u_{1}-\bar{u}_{1}\right|+\left|u_{2}-\bar{u}_{2}\right|\right) d s \\
& +C_{7} \int_{0}^{\tau}(|x-\bar{x}|+|y-\bar{y}|+|V-\bar{V}|)(s) d s .
\end{aligned}
$$

By Gronwall's inequality in integral form, we have

$$
\begin{aligned}
(|x-\bar{x}|+|y-\bar{y}|+|V-\bar{V}|)(\tau) & \leq C_{6}\left(1+C_{7} \tau e^{C_{7} \tau}\right) \int_{0}^{A}\left(\left|u_{1}-\bar{u}_{1}\right|+\left|u_{2}-\bar{u}_{2}\right|\right) d s \\
& \leq C_{6}\left(1+C_{7} A e^{C_{7} A}\right) \int_{0}^{A}\left(\left|u_{1}-\bar{u}_{1}\right|+\left|u_{2}-\bar{u}_{2}\right|\right) d s
\end{aligned}
$$

so that integrating both sides of the inequality above from $\tau=0$ to $\tau=A$, we obtain

$$
\int_{0}^{A}(|x-\bar{x}|+|y-\bar{y}|+|V-\bar{V}|) d \tau \leq C_{6} A\left(1+C_{7} A e^{C_{7} A}\right) \int_{0}^{A}\left(\left|u_{1}-\bar{u}_{1}\right|+\left|u_{2}-\bar{u}_{2}\right|\right) d s .
$$

Now, using an equivalent expression for $S$, and mimicking equation (2.17), we obtain

$$
\begin{aligned}
|S(t)-\bar{S}(t)| & =\left|c_{1} s_{1} \int_{0}^{t} e^{-m_{0}(t-\xi)} \int_{0}^{A}\left(\frac{S(\xi) V(\tau) i(\tau, \xi)}{N(\xi)}-\frac{\bar{S}(\xi) \bar{V}(\tau) \bar{i}(\tau, \xi)}{\bar{N}(\xi)}\right) d \tau d \xi\right| \\
& \leq 2 C_{8} \int_{0}^{t}\left(|S-\bar{S}|(\xi) d s+\int_{0}^{A}|i-\bar{i}|(\tau, \xi) d \tau\right) d \xi+C_{9} T \int_{0}^{A}|V(\tau)-\bar{V}(\tau)| d \tau
\end{aligned}
$$

Finally, we consider the equation for $i$ given in (2.9). Now, for $t<\tau<A$,

$$
\begin{aligned}
\int_{t}^{A}|i-\bar{i}| d \tau & =\int_{t}^{A}\left|i^{0}(\tau-t) e^{-\int_{0}^{t} m(V(\tau-t+s)) d s}-i^{0}(\tau-t) e^{-\int_{0}^{t} m(\bar{V}(\tau-t+s)) d s}\right| d \tau \\
& \leq K_{1} \int_{0}^{A}\left|i^{0}(\hat{\tau}-t)\right| \int_{0}^{A}|V(\hat{r})-\bar{V}(\hat{r})| d \hat{r} d \hat{\tau} \\
& \leq K_{1} M \int_{0}^{A}|V-\bar{V}| d \hat{\tau}
\end{aligned}
$$


where $\hat{r}=\tau-t+s, \hat{\tau}=\tau, \hat{s}=s$ and $K_{1}$ is a Lipschitz constant for the function $m$. Lastly, for $\tau<t<T$, we have

$\int_{0}^{t}\left|i_{1}-\bar{i}_{1}\right| d \tau d t$

$$
\begin{aligned}
= & \int_{0}^{t} c_{1} s_{1}\left|\frac{S(t-\tau)}{N(t-\tau)} \pi(V)(\tau) K\left(i_{1}, V\right)(t-\tau)-\frac{\bar{S}(t-\tau)}{\bar{N}(t-\tau)} \pi(\bar{V})(\tau) K\left(\bar{i}_{1}, \bar{V}\right)(t-\tau)\right| d \tau \\
\leq & \int_{0}^{t} c_{1} s_{1}\left|\frac{\pi(\tau) K(t-\tau)}{N(t-\tau)}(S(t-\tau)-\bar{S}(t-\tau))+\frac{\bar{S}(t-\tau) \bar{\pi}(\tau)}{\bar{N}(t-\tau)}(K(t-\tau)-\bar{K}(t-\tau))\right| d \tau \\
& +\int_{0}^{t} c_{1} s_{1}\left|\bar{S}(t-\tau) \frac{K(t-\tau)}{N(t-\tau)}(\pi(\tau)-\bar{\pi}(\tau))\right| d \tau \\
& +\int_{0}^{t} c_{1} s_{1}\left|\frac{\bar{S}(t-\tau)}{\bar{N}(t-\tau)} \frac{K(t-\tau)}{N(t-\tau)} \bar{\pi}(\tau)\left[\bar{S}(t-\tau)-S(t-\tau)+\int_{0}^{A}(\bar{i}(h, t-\tau)-i(h, t-\tau)) d h\right]\right| d \tau,
\end{aligned}
$$

where

$$
\pi(\tau)=e^{-\int_{0}^{\tau} m(V(s)) d s} \quad \text { and } \quad K(t-\tau)=\int_{0}^{A} V(r) i(r, t-\tau) d r .
$$

Since $0<V(\tau) \leq C$ for all $\tau>0$ (see Theorem 2.1) and $\int_{0}^{A} i(\tau, t) d \tau \leq N(t)$ a.e. $t$, we obtain $\int_{0}^{t}|i-\bar{i}|(\tau, t) d \tau$

$$
\leq 2 C_{8} \int_{0}^{t}\left(|S-\bar{S}|(\xi)+\int_{0}^{A}|i-\bar{i}|(\hat{r}, \xi) d \hat{r}\right) d \xi+C_{10} T \int_{0}^{A}|V-\bar{V}|(\hat{r}) d \hat{r},
$$

where $\xi=t-\tau$ and $\hat{r}=r$. Combining inequalities (3.10) and (3.11), we have

$$
\begin{aligned}
\int_{0}^{A}|i-\bar{i}|(\tau, t) d \tau= & \int_{0}^{t}|i-\bar{i}|(\tau, t) d \tau+\int_{t}^{A}|i-\bar{i}|(\tau, t) d \tau \\
\leq & \left(K_{1} M+C_{10} T\right) \int_{0}^{A}|V-\bar{V}|(\hat{r}) d \hat{r} \\
& +2 C_{8} \int_{0}^{t}\left(|S-\bar{S}|(\xi)+\int_{0}^{A}|i-\bar{i}|(\hat{r}, \xi) d \hat{r}\right) d \xi
\end{aligned}
$$

Next, we combine inequalities (3.9) and (3.12). This gives $|S-\bar{S}|(t)+\int_{0}^{A}|i-\bar{i}|(\tau, t) d \tau$

$$
\leq C(A, T) \int_{0}^{A}\left(\left|u_{1}-\bar{u}_{1}\right|+\left|u_{2}-\bar{u}_{2}\right|\right)(\xi) d \xi+4 C_{8} \int_{0}^{t}\left(|S-\bar{S}|(\xi)+\int_{0}^{A}|i-\bar{i}|(\hat{r}, \xi) d \hat{r}\right) d \xi
$$

Thus, by Gronwall's inequality in integral form, we obtain $|S-\bar{S}|(t)+\int_{0}^{A}|i-\bar{i}|(\tau, t) d \tau$

$$
\leq C(A, T)\left(1+4 C_{8} T e^{4 C_{8} T}\right) \int_{0}^{A}\left(\left|u_{1}-\bar{u}_{1}\right|+\left|u_{2}-\bar{u}_{2}\right|\right) d \tau .
$$

Integrating both sides of inequality (3.13) from $t=0$ to $t=T$ gives $\int_{0}^{T}|S-\bar{S}|(t) d t+\int_{0}^{T} \int_{0}^{A}|i-\bar{i}|(\tau, t) d \tau d t$

$$
\leq C(A, T)\left(1+4 C_{8} T e^{4 C_{8} T}\right) T \int_{0}^{A}\left(\left|u_{1}-\bar{u}_{1}\right|+\left|u_{2}-\bar{u}_{2}\right|\right) d \tau
$$


Finally, we combine equations (3.8) and (3.14), to have

$$
\begin{gathered}
\int_{0}^{A}(|x-\bar{x}|+|y-\bar{y}|+|V-\bar{V}|)(\tau) d \tau+\int_{0}^{T}|S-\bar{S}|(t) d t+\int_{Q}|i-\bar{i}|(\tau, t) d \tau d t \\
\leq C_{A, T} \int_{0}^{A}\left(\left|u_{1}-\bar{u}_{1}\right|+\left|u_{2}-\bar{u}_{2}\right|\right)(\tau) d \tau .
\end{gathered}
$$

where $C_{A, T}=C_{6}\left(1+C_{7} A e^{C_{7} A}\right) A+C(A, T)\left(1+4 C_{8} T e^{4 C_{8} T}\right) T$.

(ii) We find $L^{\infty}$ estimates of the state solutions by considering absolute values of $x-\bar{x}, y-\bar{y}, V-\bar{V}$ and $S-\bar{S}$, and $L^{1}$ estimate of $|i-\bar{i}|$. From equations (3.5), (3.6), (3.7) and (3.8), we have

$$
\begin{aligned}
|x-\bar{x}|(\tau) & \leq C_{1} \int_{0}^{A}\left|u_{1}-\bar{u}_{1}\right| d s+C_{2} \int_{0}^{A}(|x-\bar{x}|+|y-\bar{y}|+|V-\bar{V}|) d s \\
& \leq C_{1} \int_{0}^{A}\left|u_{1}-\bar{u}_{1}\right| d s+C_{2} C_{6} A\left(1+C_{7} A e^{C_{7} A}\right) \int_{0}^{A}\left(\left|u_{1}-\bar{u}_{1}\right|+\left|u_{2}-\bar{u}_{2}\right|\right) d s \\
|y-\bar{y}|(\tau) & \leq C_{1} \int_{0}^{A}\left|u_{1}-\bar{u}_{1}\right| d s+C_{3} C_{6} A\left(1+C_{7} A e^{C_{7} A}\right) \int_{0}^{A}\left(\left|u_{1}-\bar{u}_{1}\right|+\left|u_{2}-\bar{u}_{2}\right|\right) d s \\
|V-\bar{V}|(\tau) & \leq\left(C_{4}+C_{5} C_{6} A\left(1+C_{7} A e^{C_{7} A}\right)\right) \int_{0}^{A}\left(\left|u_{1}-\bar{u}_{1}\right|+\left|u_{2}-\bar{u}_{2}\right|\right) d s,
\end{aligned}
$$

Taking the essential supremum over all $\tau \in[0, A]$, we have

$$
\|x-\bar{x}\|_{L^{\infty}(0, A)}+\|y-\bar{y}\|_{L^{\infty}(0, A)}+\|V-\bar{V}\|_{L^{\infty}(0, A)} \leq C_{A}\left(\left\|u_{1}-\bar{u}_{1}\right\|_{L^{\infty}(0, A)}+\left\|u_{2}-\bar{u}_{2}\right\|_{L^{\infty}(0, A)}\right)
$$

Considering inequality (3.9), we have

$$
\begin{aligned}
|S-\bar{S}|(t) & \leq C_{8} \int_{0}^{T}\left(|S-\bar{S}|(\xi)+\int_{0}^{A}|i-\bar{i}|(\tau, \xi) d \tau\right) d \xi+C_{9}(T) \int_{0}^{A}|V(\tau)-\bar{V}(\tau)| d \tau \\
& \leq C_{1}(A, T) \int_{0}^{A}\left(\left|u_{1}-\bar{u}_{1}\right|+\left|u_{2}-\bar{u}_{2}\right|\right) d \tau
\end{aligned}
$$

by inequalities (3.8) and (3.14). We take the essential supremum of both sides of Inequality (3.16) over all $t \in[0, T]$. This gives

$$
\|S-\bar{S}\|_{L^{\infty}(0, T)} \leq A C_{1}(A, T)\left(\left\|u_{1}-\bar{u}_{1}\right\|_{L^{\infty}(0, A)}+\left\|u_{2}-\bar{u}_{2}\right\|_{L^{\infty}(0, A)}\right) .
$$

Lastly, to find $L^{\infty}$ estimate of $|i-\bar{i}|$, we start with the $L^{1}$ estimate of $|i-\bar{i}|$ over $\tau \in[0, A]$. Now, from equations (3.10) and (3.11), we have

$$
\begin{aligned}
\int_{0}^{A}|i-\bar{i}| d \tau & \leq 2 C_{8} \int_{0}^{t}\left(|S-\bar{S}|(\xi)+\int_{0}^{A}|i-\bar{i}|(\hat{r}, \xi) d \hat{r}\right) d \xi+\left(K_{1} M+C_{10} T\right) \int_{0}^{A}|V-\bar{V}|(\hat{r}) d \hat{r} \\
& \leq C_{3}(A, T)\left(\left\|u_{1}-\bar{u}_{1}\right\|_{L^{\infty}(0, A)}+\left\|u_{2}-\bar{u}_{2}\right\|_{L^{\infty}(0, A)}\right)
\end{aligned}
$$

by inequalities (3.8) and (3.14). Taking the essential supremum over all $t \in[0, T]$, we obtain

$$
\|i\|_{L^{\infty}\left(0, T ; L^{1}(0, A)\right)} \leq C_{3}(A, T)\left(\left\|u_{1}-\bar{u}_{1}\right\|_{L^{\infty}(0, A)}+\left\|u_{2}-\bar{u}_{2}\right\|_{L^{\infty}(0, A)}\right) .
$$

Finally, combining inequalities (3.15), (3.17) and (3.19), we obtain the desired result.

In order to characterize the optimal control pair, we differentiate the objective functional with respect to the controls. Since the objective functional is defined in terms of the state functions, we first differentiate the control-to-state map, $\left(u_{1}, u_{2}\right) \rightarrow(x, y, V, S, i)$. The derivative of the control-to-state map is called sensitivity. 
Theorem 3.3. The map $\left(u_{1}, u_{2}\right) \rightarrow(x, y, V, S, i)=(x, y, V, S, i)\left(u_{1}, u_{2}\right)$ is differentiable in the following sense:

$$
\frac{(x, y, V, S, i)\left(u_{1}+\varepsilon l_{1}, u_{2}+\varepsilon l_{2}\right)-(x, y, V, S, i)\left(u_{1}, u_{2}\right)}{\varepsilon} \rightarrow(\psi, \varphi, \phi, \theta, \omega)
$$

in $\left(L^{\infty}(\Omega)\right)^{3} \times L^{\infty}(0, T) \times L^{\infty}\left(0, T ; L^{1}(\Omega)\right)$, as $\varepsilon \rightarrow 0$ with $\left(u_{1}+\varepsilon l_{1}, u_{2}+\varepsilon l_{2}\right),\left(u_{1}, u_{2}\right) \in \mathcal{U}$ and $l_{1}, l_{2} \in$ $L^{\infty}(\Omega)$. Furthermore, the sensitivity functions satisfy

$$
\begin{aligned}
\frac{d \psi}{d \tau}= & -\beta_{1}\left(1-u_{1}\right) V \psi-\beta_{1}\left(1-u_{1}\right) x \phi-\mu \psi+\beta_{1} l_{1} V x \\
\frac{d \varphi}{d \tau}= & \beta_{1}\left(1-u_{1}\right) V \psi-d_{1} \varphi+\beta_{1}\left(1-u_{1}\right) x \phi-\beta_{1} l_{1} V x \\
\frac{d \phi}{d \tau}= & -\hat{\beta}_{1}\left(1-u_{1}\right) V \psi+\nu_{1}\left(1-u_{2}\right) d_{1} \varphi-\left(\delta_{1}+s_{1}+\hat{\beta}_{1}\left(1-u_{1}\right) x\right) \phi \\
& +\hat{\beta}_{1} l_{1} V x-\nu_{1} d_{1} l_{2} y \\
\frac{d \theta}{d t}= & -m_{0} \theta(t)-\frac{c_{1} s_{1}}{N(t)}\left(1-\frac{S(t)}{N(t)}\right) \theta(t) \int_{\Omega} i(\tau, t) V(\tau) d \tau \\
& -\frac{c_{1} s_{1} S(t)}{N(t)} \int_{\Omega} V(\tau) \omega(\tau, t) d \tau-\frac{c_{1} s_{1} S(t)}{N(t)} \int_{\Omega} i(\tau, t) \phi(\tau) d \tau \\
& +\frac{c_{1} s_{1} S(t)}{N(t)^{2}} \int_{\Omega} i(\tau, t) V(\tau) \int_{\Omega} \omega(h, t) d h d \tau \quad i n \quad(0, T) \\
\frac{\partial \omega}{\partial t}+ & \frac{\partial \omega}{\partial \tau}=-m(V) \omega-m^{\prime}(V) \phi i \quad i n \quad \Omega \times(0, T)
\end{aligned}
$$

with initial and boundary conditions

$$
\psi(0)=0, \quad \varphi(0)=0, \quad \phi(0)=0, \quad \theta(0)=0, \quad \omega(\tau, 0)=0, \forall \tau \in \Omega=(0, A)
$$

and

$$
\begin{aligned}
\omega(0, t) & =\frac{c_{1} s_{1}}{N(t)}\left(1-\frac{S(t)}{N(t)}\right) \theta(t) \int_{\Omega} i(\tau, t) V(\tau) d \tau+\frac{c_{1} s_{1} S(t)}{N(t)} \int_{\Omega} V(\tau) \omega(\tau, t) d \tau \\
& +\frac{c_{1} s_{1} S(t)}{N(t)} \int_{\Omega} i(\tau, t) \phi(\tau) d \tau-\frac{c_{1} s_{1} S(t)}{N(t)^{2}} \int_{\Omega} i(\tau, t) V(\tau) \int_{\Omega} \omega(h, t) d h d \tau
\end{aligned}
$$

Proof. Since the map $\left(u_{1}, u_{2}\right) \rightarrow(x, y, V, S, i)$ is Lipschitz in $L^{\infty}$, we have the existence of the Gâteaux derivatives (or sensitivities) $\psi, \varphi, \phi, \theta$ and $\omega$ by Barbu [7, p. 17] and Fister et al. [19,20]. Passing to the limit in the representation of the quotients, gives that $\psi, \varphi, \phi, \theta$ and $\omega$ satisfy system (3.20)-(3.26).

We divide the sensitivity equations in Theorem 3.3 into three operators, depending on the independent variables on five components. These operators will be used in deriving a characterization for the controls $u_{1}$ and $u_{2}$. The three sensitivity operators, $\mathcal{L}_{1}, \mathcal{L}_{2}$ and $\mathcal{L}_{3}$, and the corresponding sensitivity equations are:

$$
\mathcal{L}_{1}\left[\begin{array}{l}
\psi \\
\varphi \\
\phi
\end{array}\right]=\left[\begin{array}{c}
\beta_{1} l_{1} V x \\
-\beta_{1} l_{1} V x \\
\hat{\beta}_{1} l_{1} V x-\nu_{1} d_{1} l_{2} y
\end{array}\right], \quad \mathcal{L}\left[\begin{array}{c}
\theta \\
\omega
\end{array}\right] \equiv\left[\begin{array}{c}
\mathcal{L}_{2} \theta \\
\mathcal{L}_{3} \omega
\end{array}\right]=\left[\begin{array}{c}
0 \\
0
\end{array}\right]
$$

where 


$$
\begin{aligned}
& \mathcal{L}_{1}\left[\begin{array}{c}
\psi \\
\varphi \\
\phi
\end{array}\right]=\left[\begin{array}{l}
L_{1} \psi \\
L_{1} \varphi \\
L_{1} \phi
\end{array}\right]+M\left[\begin{array}{l}
\psi \\
\varphi \\
\phi
\end{array}\right], \quad\left[\begin{array}{l}
L_{1} \psi \\
L_{1} \varphi \\
L_{1} \phi
\end{array}\right]=\left[\begin{array}{c}
\frac{d \psi}{d \tau} \\
\frac{d \varphi}{d \tau} \\
\frac{d \phi}{d \tau}
\end{array}\right] \\
& \mathcal{L}\left[\begin{array}{c}
\theta \\
\omega
\end{array}\right]=\left[\begin{array}{c}
L_{2} \theta \\
L_{3} \omega
\end{array}\right]+N\left[\begin{array}{c}
\theta \\
\omega
\end{array}\right], \quad\left[\begin{array}{c}
L_{2} \theta \\
L_{3} \omega
\end{array}\right]=\left[\begin{array}{c}
\frac{d \theta}{d t} \\
\frac{\partial \omega}{\partial t}+\frac{\partial \omega}{\partial \tau}
\end{array}\right] \\
& M=\left(\begin{array}{ccc}
\beta_{1}\left(1-u_{1}\right) V+\mu & 0 & \beta_{1}\left(1-u_{1}\right) x \\
-\beta_{1}\left(1-u_{1}\right) V & d_{1} & -\beta_{1}\left(1-u_{1}\right) x \\
\hat{\beta}_{1}\left(1-u_{1}\right) V & -d_{1} \nu_{1}\left(1-u_{2}\right) & \delta_{1}+s_{1}+\hat{\beta}_{1}\left(1-u_{1}\right) x
\end{array}\right), \\
& N\left[\begin{array}{c}
\theta \\
\omega
\end{array}\right]=\left(\begin{array}{c}
B(\phi, \theta, \omega)+C(\omega)+m_{0} \theta \\
m^{\prime}(V) \phi+m\left(V_{1}\right) \omega
\end{array}\right) \\
& B(\phi, \theta, \omega)=\frac{c_{1} s_{1}}{N(t)}\left(1-\frac{S(t)}{N(t)}\right) \theta(t) \int_{\Omega} i(\tau, t) V(\tau) d \tau+\frac{c_{1} s_{1} S(t)}{N(t)} \int_{\Omega} V(\tau) \omega(\tau, t) d \tau \\
& +\frac{c_{1} s_{1} S(t)}{N(t)} \int_{\Omega} i(\tau, t) \phi(\tau) d \tau \\
& C(\omega)=-\frac{c_{1} s_{1} S(t)}{N(t)^{2}} \int_{\Omega} i(\tau, t) V(\tau) \int_{\Omega} \omega(h, t) d h d \tau
\end{aligned}
$$

We derive the adjoint system from the sensitivity equations. Thus, if $\lambda, \xi, \eta, p$, and $q$ are adjoint variables, then we find adjoint operators $\mathcal{L}_{j}^{*}$, for $j=1,2,3$ such that

$$
\begin{aligned}
& \int_{\Omega}(\lambda, \xi, \eta) \mathcal{L}_{1}(\psi, \varphi, \phi) d \tau+\int_{0}^{T} p \mathcal{L}_{2} \theta d t+\int_{Q} q_{1} \mathcal{L}_{3} \omega d \tau d t \\
&=\int_{\Omega}(\psi, \varphi, \phi) \mathcal{L}_{1}^{*}(\lambda, \xi, \eta) d \tau+\int_{0}^{T} \theta \mathcal{L}_{2}^{*} p d t+\int_{Q} \omega \mathcal{L}_{3}^{*} q d \tau d t
\end{aligned}
$$

with adjoint equations (in the weak sense defined below)

$$
\mathcal{L}_{1}^{*}\left[\begin{array}{c}
\lambda \\
\xi \\
\eta
\end{array}\right]=\left[\begin{array}{c}
0 \\
0 \\
\int_{0}^{T} A_{1} i(\tau, t) d t
\end{array}\right], \quad \mathcal{L}^{*}\left[\begin{array}{l}
p \\
q
\end{array}\right]=\left[\begin{array}{c}
0 \\
A_{1} V+A_{2} u_{1}+A_{3} u_{2}
\end{array}\right]
$$

and

$$
\mathcal{L}^{*}\left[\begin{array}{c}
p \\
q
\end{array}\right] \equiv\left[\begin{array}{c}
\mathcal{L}_{2}^{*} p \\
\mathcal{L}_{3}^{*} q
\end{array}\right] .
$$

The right-hand side of the adjoint equations (3.29) are obtained by differentiating the integrand of the objective functional (3.4) with respect to each state variable. The transversality conditions associated with the adjoint variables are:

$$
\begin{aligned}
\lambda(A) & =0, \quad \xi(A)=0, \quad \eta(A)=0, \quad p(T)=0 \\
q(\tau, T) & =0, \quad \text { for } \quad \tau \in \Omega \\
q(A, t) & =0, \quad \text { for } \quad t \in(0, T) .
\end{aligned}
$$

From the sensitivity system in Theorem 3.3 and the relationship between the sensitivity and adjoint operators given by equation (3.28), we use integration by parts to throw the derivatives on the differential operators in the sensitivity functions $\psi, \varphi, \phi, \eta, \theta$, and $\omega$ onto the adjoint functions $\lambda, \xi, \eta, p$ and $q[39]$. Applying the initial and boundary conditions in equations (3.25)-(3.26), and the final time conditions 
in (3.30)-(3.32), we have the following adjoint system corresponding to controls $\left(u_{1}, u_{2}\right)$ and states $(x, y, V, S, i)=(x, y, V, S, i)\left(u_{1}, u_{2}\right)$ :

$$
\begin{aligned}
-\frac{d \lambda}{d \tau}= & -\left(\beta_{1}\left(1-u_{1}\right) V+\mu\right) \lambda+\beta_{1}\left(1-u_{1}\right) V \xi-\hat{\beta}_{1}\left(1-u_{1}\right) V \eta \\
-\frac{d \xi}{d \tau}= & -d_{1} \xi+\nu_{1}\left(1-u_{2}\right) d_{1} \eta \\
-\frac{d \eta}{d \tau}= & -\beta_{1}\left(1-u_{1}\right) x \lambda+\beta_{1}\left(1-u_{1}\right) \xi-\left(\delta_{1}+s_{1}+\hat{\beta}_{1}\left(1-u_{1}\right) x\right) \eta \\
& -c_{1} s_{1} \int_{0}^{T} \frac{S(t) i(\tau, t)}{N(t)}(p(t)-q(0, t)) d t-m^{\prime}(V) \int_{0}^{T} i(\tau, t) q(\tau, t) d t \\
& +\int_{0}^{T} A_{1} i(\tau, t) d t \\
-\frac{d p}{d t}= & -m_{0} p-\frac{c_{1} s_{1}}{N}(p-q(0, t))\left(1-\frac{S}{N}\right) \int_{0}^{A} V(\tau) i(\tau, t) d \tau \\
-\frac{\partial q}{\partial t}-\frac{\partial q}{\partial \tau}= & -m(V) q+\frac{c_{1} s_{1} S}{N^{2}}(p-q(0, t)) \int_{0}^{A} V(\tau) i(\tau, t) d \tau-c_{1} s_{1}(p-q(0, t)) \frac{S V}{N} \\
& +A_{1} V+A_{2} u_{1}+A_{3} u_{2},
\end{aligned}
$$

with final time conditions (3.30)-(3.32). Given the sensitivity and adjoint equations, we state a theorem that characterizes the weak solution to our problem.

Theorem 3.4. (Weak Solution) The weak solution of the adjoint system satisfies

$$
\int_{\Omega}\left(\lambda \alpha_{1}+\xi \alpha_{2}+\eta \alpha_{3}-g \int_{0}^{T} A_{1} i(\tau, t) d t\right) d \tau-\int_{0}^{T} \int_{0}^{A}\left(A_{1} V+A_{2} u_{1}+A_{3} u_{2}\right) n d \tau d t=0
$$

where $\alpha_{1}, \alpha_{2}, \alpha_{3}$ are $L^{\infty}(0, A)$ functions obtained from test functions $z, f$ and $g$, and $r$ and $n$ satisfy equations (3.36) and (3.37) such that

$$
\begin{aligned}
& \frac{d z}{d \tau}+\beta_{1}\left(1-u_{1}\right) V z+\beta_{1}\left(1-u_{1}\right) x g+\mu z=\alpha_{1} \\
& \frac{d f}{d \tau}-\beta_{1}\left(1-u_{1}\right) V z-\beta_{1}\left(1-u_{1}\right) x g+d_{1} f=\alpha_{2} \\
& \frac{d g}{d \tau}+\hat{\beta}_{1}\left(1-u_{1}\right) V z-\nu_{1}\left(1-u_{2}\right) d_{1} f+\left(\delta_{1}+s_{1}+\hat{\beta}_{1}\left(1-u_{1}\right) x\right) g=\alpha_{3} \\
& \frac{d r}{d t}+m_{0} r+\frac{c_{1} s_{1}}{N}\left(1-\frac{S}{N}\right) r \int_{0}^{A} i(\tau, t) V(\tau) d \tau+\frac{c_{1} s_{1} S}{N} \int_{0}^{A} V(\tau) n(\tau, t) d \tau \\
& +\frac{c_{1} s_{1} S}{N} \int_{0}^{A} g(\tau) i(\tau, t) d \tau-\frac{c_{1} s_{1} S}{N^{2}} \int_{0}^{A} i(\tau, t) V(\tau) \int_{0}^{A} n(h, t) d h d \tau=0 \\
& \frac{\partial n(\tau, t)}{\partial t}+\frac{\partial n(\tau, t)}{\partial \tau}+m(V) n+m^{\prime}(V) g i=0 \quad \text { in } Q
\end{aligned}
$$

with initial and boundary conditions

$$
z(0)=0, \quad f(0)=0, \quad g(0)=0, \quad r(0)=0, \quad n(\tau, 0)=0 \quad \text { for } \quad \tau \in(0, A)
$$

and

$$
\begin{aligned}
n(0, t)= & \frac{c_{1} s_{1}}{N}\left(1-\frac{S}{N}\right) r \int_{0}^{A} i(\tau, t) V(\tau) d \tau+\frac{c_{1} s_{1} S}{N} \int_{0}^{A} V(\tau) n(\tau, t) d \tau \\
& +\frac{c_{1} s_{1} S}{N} \int_{0}^{A} g(\tau) i(\tau, t) d \tau-\frac{c_{1} s_{1} S}{N^{2}} \int_{0}^{A} i(\tau, t) V(\tau) \int_{0}^{A} n(h, t) d h d \tau .
\end{aligned}
$$


Proof. Follows from the sensitivity equations and adjoint system, with $\alpha_{1}=\beta_{1} l_{1} V x$, $\alpha_{2}=-\beta_{1} l_{1} V x$ and $\alpha_{3}=\hat{\beta_{1}} l_{1} V x-\nu_{1} d_{1} l_{2} y$.

We establish the existence of solution to the adjoint system via the existence of solution $(z, f, g, r, n)$ to system (3.38)-(3.44) (see Barbu [7], Fister and Lenhart [19,20]). The solution of the adjoint system satisfies a Lipschitz property analogous to Theorem 3.2. This property will be used in proving uniqueness of an optimal control pair.

Theorem 3.5. For $\left(u_{1}, u_{2}\right) \in \mathcal{U}$, the adjoint system (3.33)-(3.37) has a weak solution $(\lambda, \xi, \eta, p, q)$ in $\left(L^{\infty}(0, A)\right)^{3} \times L^{\infty}(0, T) \times L^{\infty}\left(0, T, L^{1}(0, A)\right)$ such that

$$
\begin{aligned}
& \|\lambda-\bar{\lambda}\|_{L^{\infty}(\Omega)}+\|\xi-\bar{\xi}\|_{L^{\infty}(\Omega)}+\|\eta-\bar{\eta}\|_{L^{\infty}(\Omega)}+\|p-\bar{p}\|_{L^{\infty}(0, T)}+\|q-\bar{q}\|_{L^{\infty}(Q)} \\
& \leq \hat{C}_{A, T}\left(\left\|u_{1}-\bar{u}_{1}\right\|_{L^{\infty}(\Omega)}+\left\|u_{2}-\bar{u}_{2}\right\|_{L^{\infty}(\Omega)}\right) .
\end{aligned}
$$

Proof. Follows like in Theorem 3.2, part (ii).

\subsection{Characterization of Optimal Control}

We use the Ekeland's Principle $[3,13]$ to characterize optimal control of first-order PDEs. To do this, we embed the objective functional $J$ in the space $L^{1}(\Omega) \times L^{1}(Q)$ by defining $[6,19,20]$

$$
\mathcal{J}\left(u_{1}, u_{2}\right)= \begin{cases}J\left(u_{1}, u_{2}\right) & \text { if }\left(u_{1}, u_{2}\right) \in \mathcal{U} \\ +\infty & \text { if }\left(u_{1}, u_{2}\right) \notin \mathcal{U}\end{cases}
$$

In order to characterize the optimal control pair, we differentiate the objective functional with respect to the controls. However, since the objective functional is a function of the state functions, we must differentiate the state functions with respect to the controls.

Theorem 3.6. If $\left(u_{1}^{*}, u_{2}^{*}\right) \in \mathcal{U}$ is an optimal control pair minimizing (3.45), and $\left(x^{*}, y^{*}, V^{*}, S^{*}, i^{*}\right)$ and $(\lambda, \xi, \eta, p, q)$ are the corresponding state and adjoint solutions, then

$$
\begin{aligned}
& u_{1}^{*}(\tau)=\mathcal{F}_{1}\left(\frac{\beta_{1} V^{*} x^{*}(\xi-\lambda)-\hat{\beta}_{1} V^{*} x^{*} \eta-A_{2} \int_{0}^{T} i^{*}(\tau, t) d t}{2 B}\right) \text { a.e. in } L^{1}(\Omega) \\
& u_{2}^{*}(\tau)=\mathcal{F}_{2}\left(\frac{\nu_{1} d_{1} \eta y^{*}-A_{3} \int_{0}^{T} i^{*}(\tau, t) d t}{2 B}\right) \quad \text { a.e. in } L^{1}(\Omega),
\end{aligned}
$$

where

$$
\mathcal{F}_{j}(x)=\left\{\begin{array}{l}
0, \quad x<0 \\
x, 0 \leq x \leq \tilde{u}_{j} \quad \text { for } \quad j=1,2 \\
\tilde{u}_{j}, \quad x>\tilde{u}_{j}
\end{array}\right.
$$

Proof. Since $\left(u_{1}^{*}, u_{2}^{*}\right)$ is an optimal control pair and we seek to minimize our functional, we have

$$
0 \leq \lim _{\varepsilon \rightarrow 0^{+}} \frac{\mathcal{J}\left(u_{1}^{*}+\varepsilon l_{1}, u_{2}^{*}+\varepsilon l_{2}\right)-\mathcal{J}\left(u_{1}^{*}, u_{2}^{*}\right)}{\varepsilon}
$$




$$
\begin{aligned}
= & \lim _{\varepsilon \rightarrow 0^{+}} \int_{0}^{T} \int_{0}^{A}\left(A_{1} V^{\varepsilon}\left(\frac{i^{\varepsilon}-i^{*}}{\varepsilon}\right)+A_{1} i^{*}\left(\frac{V^{\varepsilon}-V^{*}}{\varepsilon}\right)+\frac{A_{2}\left(i^{\varepsilon} u_{1}^{\varepsilon}-i^{*} u_{1}^{*}\right)}{\varepsilon}\right) d \tau d t \\
& +\lim _{\varepsilon \rightarrow 0^{+}} \int_{0}^{T} \int_{0}^{A}\left(\frac{A_{3}\left(i^{\varepsilon} u_{2}^{\varepsilon}-i^{*} u_{2}^{*}\right)}{\varepsilon}\right)+\lim _{\varepsilon \rightarrow 0^{+}} \int_{0}^{A} B\left(\frac{\left(u_{1}^{\varepsilon}\right)^{2}-\left(u_{1}^{*}\right)^{2}}{\varepsilon}+\frac{\left(u_{2}^{\varepsilon}\right)^{2}-\left(u_{2}^{*}\right)^{2}}{\varepsilon}\right) d \tau \\
= & \int_{0}^{A}(\psi, \varphi, \phi)\left(\begin{array}{c}
0 \\
0 \\
\int_{0}^{T} A_{1} i^{*}(\tau, t) d t
\end{array}\right) d \tau+\int_{0}^{T} \theta .0 d t+2 B \int_{0}^{A}\left(l_{1} u_{1}^{*}+l_{2} u_{2}^{*}\right) d \tau \\
& +\int_{0}^{T} \int_{0}^{A} \omega\left(A_{1} V^{*}+A_{2} u_{1}^{*}+A_{3} u_{2}^{*}+l_{1} A_{2} i^{*}+l_{2} A_{3} i^{*}\right) d \tau d t \\
= & \int_{\Omega}(\lambda, \xi, \eta) \mathcal{L}_{1}\left(\begin{array}{l}
\psi \\
\varphi \\
\phi
\end{array}\right) d \tau+\int_{0}^{T} p \mathcal{L}_{2} \theta d t+2 B \int_{0}^{A}\left(l_{1} u_{1}^{*}+l_{2} u_{2}^{*}\right) d \tau \\
& +\int_{0}^{T} \int_{0}^{A}\left(q \mathcal{L}_{3} \omega+l_{1} A_{2} i^{*}+l_{2} A_{3} i^{*}\right) d \tau d t,
\end{aligned}
$$

in an appropriate weak sense. Using the sensitivity operators, we have

$$
\begin{aligned}
0 \leq & \int_{0}^{A}\left((\lambda, \xi, \eta)\left(\begin{array}{c}
\beta_{1} l_{1} V^{*} x^{*} \\
-\beta_{1} l_{1} V^{*} x^{*} \\
\hat{\beta}_{1} l_{1} V^{*} x^{*}-\nu_{1} d_{1} l_{2} y^{*}
\end{array}\right)+2 B\left(l_{1} u_{1}^{*}+l_{2} u_{2}^{*}\right)\right) d \tau \\
& +\int_{0}^{T} \int_{0}^{A}\left(A_{2} l_{1} i^{*}(\tau, t)+A_{3} l_{2} i^{*}(\tau, t)\right) d \tau d t \\
= & \int_{0}^{A} l_{1}\left(\beta_{1} V^{*} x^{*}(\lambda-\xi)+\hat{\beta}_{1} V^{*} x^{*} \eta+2 B u_{1}^{*}+A_{2} \int_{0}^{T} i^{*}(\tau, t) d t\right) d \tau \\
& +\int_{0}^{A} l_{2}\left(2 B u_{2}^{*}-\nu_{1} d_{1} y^{*} \eta+A_{3} \int_{0}^{T} i^{*}(\tau, t) d t\right) d \tau .
\end{aligned}
$$

By standard arguments, varying $l_{1}$ and $l_{2}$, we obtain the characterization given in equations (3.46) and (3.47).

\subsection{Existence of Optimal Control Pair}

The lower semicontinuity of the functional, $\mathcal{J}$, defined in equation (3.45) with respect to $L^{1}$ convergence is needed to prove the existence of optimal control pair. Since solutions of first-order partial differential equations are known for nonsmoothness, the objective functional is not weakly lower semicontinuous with respect to $L^{1}$. Thus, existence results for an optimal control are not guaranteed [13]. Therefore, we circumvent this by applying the following Ekeland's Variational Principle, which guarantees the existence of a minimizing sequence: For $\varepsilon>0$, there exist $\left(u_{1}^{\varepsilon}, u_{2}^{\varepsilon}\right) \in L^{1}(0, A) \times L^{1}(0, A)$ such that

$$
\begin{aligned}
\text { (i) } \mathcal{J}\left(u_{1}^{\varepsilon}, u_{2}^{\varepsilon}\right) & \leq \inf _{\left(u_{1}, u_{2}\right) \in \mathcal{U}} \mathcal{J}\left(u_{1}, u_{2}\right)+\varepsilon \\
\text { (ii) } \mathcal{J}\left(u_{1}^{\varepsilon}, u_{2}^{\varepsilon}\right) & =\min _{\left(u_{1}, u_{2}\right) \in \mathcal{U}} \mathcal{J}_{\varepsilon}\left(u_{1}, u_{2}\right), \\
\text { where } \mathcal{J}_{\varepsilon}\left(u_{1}, u_{2}\right) & =\mathcal{J}\left(u_{1}, u_{2}\right)+\sqrt{\varepsilon}\left(\left\|u_{1}^{\varepsilon}-u_{1}\right\|_{L^{1}(0, A)}+\left\|u_{2}^{\varepsilon}-u_{2}\right\|_{L^{1}(0, A)}\right) .
\end{aligned}
$$

We shall show that the minimizer, $\left(u_{1}^{\varepsilon}, u_{2}^{\varepsilon}\right)$, of the approximate functional converges to the optimal controls $\left(u_{1}^{*}, u_{2}^{*}\right)$ in $L^{\infty}(0, A) \times L^{\infty}(0, A)$. We start by proving the lower semicontinuity of the functional $\mathcal{J}$.

\section{Theorem 3.7. (Lower semicontinuity)}

The functional $\mathcal{J}: L^{1}(\Omega) \times L^{1}(\Omega) \rightarrow(-\infty,+\infty]$ is lower semicontinuous 
Proof. Let $\left(u_{1}^{n}, u_{2}^{n}\right) \rightarrow\left(u_{1}, u_{2}\right)$ in $L^{1}(0, A) \times L^{1}(0, A)$, and assume that $(x, y, V, S, i)$ is the state solution corresponding to $\left(u_{1}, u_{2}\right)$ and $\left(x^{n}, y^{n}, V^{n}, S^{n}, i^{n}\right)$ is the state solution corresponding to $\left(u_{1}^{n}, u_{2}^{n}\right)$, then by Theorem 3.2, part (i), we have

$$
\begin{gathered}
x^{n} \rightarrow x, \quad y^{n} \rightarrow y, \quad V^{n} \rightarrow V \quad \text { in } \quad L^{1}(0, A) \\
S^{n} \rightarrow S \quad \text { in } \quad L^{1}(0, T), \quad \text { and } \quad i^{n} \rightarrow i \quad \text { in } \quad L^{1}((0, A) \times(0, T)) .
\end{gathered}
$$

Thus, on a subsequence, denoted by itself, we have

$$
\begin{gathered}
u_{1}^{n} \rightarrow u_{1}, \quad u_{2}^{n} \rightarrow u_{2}, \quad x^{n} \rightarrow x, \quad y^{n} \rightarrow y, \quad V^{n} \rightarrow V \text { a.e. in }(0, A) \\
S^{n} \rightarrow S \quad \text { a.e. } \quad \text { in } \quad(0, T), \quad \text { and } \quad i^{n} \rightarrow i \quad \text { a.e. } \quad \text { in } \quad(0, A) \times(0, T),
\end{gathered}
$$

by Theorem 5, p. 21 [15]. Hence, on a subsequence, we have

$$
\left(u_{1}^{n}\right)^{2} \rightarrow\left(u_{1}\right)^{2} \quad \text { and } \quad\left(u_{2}^{n}\right)^{2} \rightarrow\left(u_{2}\right)^{2} \quad \text { a.e. in }(0, A),
$$

and

$$
A_{1} i^{n} V^{n}+i^{n}\left(A_{2} u_{1}^{n}+A_{3} u_{2}^{n}\right) \rightarrow A_{1} i V+i\left(A_{2} u_{1}+A_{3} u_{2}\right) \quad \text { in } \quad(0, A) \times(0, T),
$$

by Lemma 3.4.3, p. 100 [3]. Using Fatou's Lemma [15], we have that on a subsequence, $\int_{0}^{T} \int_{0}^{A}\left(A_{1} i(\tau, t) V(\tau)+i(\tau, t)\left(A_{2} u_{1}+A_{3} u_{2}\right)\right) d \tau d t$

$$
\begin{aligned}
& =\int_{0}^{T} \int_{0}^{A} \liminf _{n \rightarrow \infty}\left(A_{1} i^{n} V^{n}+i^{n}\left(A_{2} u_{1}^{n}+A_{3} u_{2}^{n}\right)\right) d \tau d t \\
& \leq \liminf _{n \rightarrow \infty} \int_{0}^{T} \int_{0}^{A}\left(A_{1} i^{n} V^{n}+i^{n}\left(A_{2} u_{1}^{n}+A_{3} u_{2}^{n}\right)\right) d \tau d t
\end{aligned}
$$

and

$$
\begin{aligned}
\int_{0}^{A} B\left(\left(u_{1}\right)^{2}+\left(u_{2}\right)^{2}\right) d \tau & =B \int_{0}^{A} \liminf _{n \rightarrow \infty}\left(\left(u_{1}^{n}\right)^{2}+\left(u_{2}^{n}\right)^{2}\right) d \tau \\
& \leq \liminf _{n \rightarrow \infty} \int_{0}^{A} B\left(\left(u_{1}^{n}\right)^{2}+\left(u_{2}^{n}\right)^{2}\right) d \tau
\end{aligned}
$$

Combining equations (3.49) and (3.50), we have

$$
\begin{aligned}
\mathcal{J}\left(u_{1}, u_{2}\right) & =\int_{0}^{T} \int_{0}^{A}\left(A_{1} i V+i\left(A_{2} u_{1}+A_{3} u_{2}\right)\right) d \tau d t+B \int_{0}^{A}\left(\left(u_{1}\right)^{2}+\left(u_{2}\right)^{2}\right) d \tau \\
& \leq \lim _{n \rightarrow \infty} \inf \mathcal{J}\left(u_{1}^{n}, u_{2}^{n}\right) .
\end{aligned}
$$

Hence, the functional $\mathcal{J}$ is lower semicontinuous.

Theorem 3.8. If $\left(u_{1}^{\varepsilon}, u_{2}^{\varepsilon}\right)$ is an optimal control pair minimizing the approximate functional, $\mathcal{J}_{\mathcal{\varepsilon}}$, then $\left(u_{1}^{\varepsilon}, u_{1}^{\varepsilon}\right)$

$$
=\mathcal{F}\left(\frac{\beta_{1} V^{\varepsilon} x^{\varepsilon}\left(\xi^{\varepsilon}-\lambda^{\varepsilon}\right)-\hat{\beta_{1}} V^{\varepsilon} x^{\varepsilon} \eta^{\varepsilon}-A_{2} K^{\varepsilon}(\tau)-\sqrt{\varepsilon} \kappa_{1}^{\varepsilon}}{2 B}, \frac{\nu_{1} d_{1} \eta y^{\varepsilon}-A_{3} K^{\varepsilon}(\tau)-\sqrt{\varepsilon} \kappa_{2}^{\varepsilon}}{2 B}\right),
$$

where $K^{\varepsilon}(\tau)=\int_{0}^{T} i^{\varepsilon}(\tau, t) d t$, and the functions $\kappa_{1}, \kappa_{2} \in L^{\infty}(0, A)$, with $\left|\kappa_{1}(\tau)\right|=1$ and $\left|\kappa_{2}(\tau)\right|=1$, for all $\tau \in(0, A)$. 
Proof. Since $\left(u_{1}^{\varepsilon}, u_{2}^{\varepsilon}\right)$ is an optimal control pair minimizing the approximate functional $\mathcal{J}_{\varepsilon}$,

$$
\begin{aligned}
0 \leq & \lim _{\alpha \rightarrow 0^{+}} \frac{\mathcal{J}_{\varepsilon}\left(u_{1}^{\varepsilon}+\alpha l_{1}^{\varepsilon}, u_{2}^{\varepsilon}+\alpha l_{2}^{\varepsilon}\right)-\mathcal{J}_{\varepsilon}\left(u_{1}^{\varepsilon}, u_{2}^{\varepsilon}\right)}{\alpha} \\
= & \lim _{\alpha \rightarrow 0^{+}} \frac{\mathcal{J}\left(u_{1}^{\varepsilon}+\alpha l_{1}^{\varepsilon}, u_{2}^{\varepsilon}+\alpha l_{2}^{\varepsilon}\right)-\mathcal{J}\left(u_{1}^{\varepsilon}, u_{2}^{\varepsilon}\right)}{\alpha}+\sqrt{\varepsilon}\left(\left\|l_{1}^{\varepsilon}\right\|_{L^{1}(0, A)}+\left\|l_{2}^{\varepsilon}\right\|_{L^{1}(0, A)}\right) \\
= & \int_{0}^{A} l_{1}^{\varepsilon}\left(\beta_{1} V^{\varepsilon} x^{\varepsilon}\left(\lambda^{\varepsilon}-\xi^{\varepsilon}\right)+\hat{\beta}_{1} V^{\varepsilon} x^{\varepsilon} \eta^{\varepsilon}+2 B u_{1}^{\varepsilon}+A_{2} \int_{0}^{T} i^{\varepsilon}(\tau, t) d t+\sqrt{\varepsilon} \kappa_{1}^{\varepsilon}\right) d \tau \\
& +\int_{0}^{A} l_{2}^{\varepsilon}\left(2 B u_{2}^{\varepsilon}-\nu_{1} d_{1} y^{\varepsilon} \eta^{\varepsilon}+A_{3} \int_{0}^{T} i^{\varepsilon}(\tau, t) d t+\sqrt{\varepsilon} \kappa_{2}^{\varepsilon}\right) d \tau
\end{aligned}
$$

where $\kappa_{j}^{\varepsilon}=\frac{\left|l_{j}^{\varepsilon}\right|}{l_{j}^{\varepsilon}} \in L^{\infty}(0, A)$ for $j=1,2$, with $\left|\kappa_{j}^{\varepsilon}\right|=1$, and using equation (3.48) in

Theorem 3.6. By standard optimal control arguments (see Theorem 3.6), we have the desired result.

\subsection{Uniqueness of Optimal Control Pair}

In this subsection, we establish uniqueness of optimal control pair, by using the Lipschitz properties of the state and adjoint solutions given in Theorems 3.2 and 3.5, respectively, as well as the minimizing sequence obtained from the Ekeland's Variational Principle. Finally, we shall show that the minimizer, $\left(u_{1}^{\varepsilon}, u_{2}^{\varepsilon}\right)$, of the approximate functional, $\mathcal{J}_{\varepsilon}$, converges to the optimal control, $\left(u_{1}^{*}, u_{2}^{*}\right)$.

Theorem 3.9. (Uniqueness) If $\frac{A}{2 B}$ is sufficiently small, then there exists a unique optimal control pair $\left(u_{1}^{*}, u_{2}^{*}\right) \in \mathcal{U}$ minimizing the objective functional $\mathcal{J}$.

Proof. Let $\mathcal{F}(x, y)=\left(\mathcal{F}_{1}(x), \mathcal{F}_{2}(y)\right)$ and define $L: \mathcal{U} \rightarrow \mathcal{U}$, such that

$$
L\left(u_{1}, u_{2}\right)=\mathcal{F}\left(\frac{\beta_{1} V x(\xi-\lambda)-\hat{\beta}_{1} V x \eta-A_{2} K(\tau)}{2 B}, \frac{\nu_{1} d_{1} \eta y-A_{3} K(\tau)}{2 B}\right),
$$

where $K(\tau)=\int_{0}^{T} i(\tau, t) d t$, and $(x, y, V, S, i)$ and $(\lambda, \xi, \eta, p, q)$ are the state and adjoint solutions corresponding to the control pair $\left(u_{1}, u_{2}\right)$. Using the Lipschitz properties of the state and adjoint systems in Theorems 3.2 and 3.5, respectively, we have

$$
\begin{aligned}
\| L\left(u_{1}, u_{2}\right) & -L\left(\bar{u}_{1}, \bar{u}_{2}\right)\|\equiv\| \mathcal{F}_{1}\left(u_{1}\right)-\mathcal{F}_{1}\left(\bar{u}_{1}\right)\left\|_{L^{\infty}(0, A)}+\right\| \mathcal{F}_{2}\left(u_{2}\right)-\mathcal{F}_{2}\left(\bar{u}_{2}\right) \|_{L^{\infty}(0, A)} \\
\leq & \left\|\frac{\beta_{1} V x(\xi-\lambda)-\hat{\beta}_{1} V x \eta-A_{2} K(\tau)}{2 B}-\frac{\beta_{1} \bar{V} \bar{x}(\bar{\xi}-\bar{\lambda})-\hat{\beta}_{1} \bar{V} \bar{x} \bar{\eta}-A_{2} \bar{K}(\tau)}{2 B}\right\|_{L^{\infty}(0, A)} \\
& +\left\|\frac{\nu_{1} d_{1} \eta y-A_{3} \int_{0}^{T} i(\tau, t) d t}{2 B}-\frac{\nu_{1} d_{1} \bar{\eta} \bar{y}-A_{3} \int_{0}^{T} \bar{i}(\tau, t) d t}{2 B}\right\|_{L^{\infty}(0, A)} \\
\leq & \frac{C A}{2 B}\left(\left\|u_{1}-\bar{u}_{1}\right\|_{L^{\infty}(0, A)}+\left\|u_{2}-\overline{u_{2}}\right\|_{L^{\infty}(0, A)}\right) .
\end{aligned}
$$

Thus,

$$
\left\|L\left(u_{1}, u_{2}\right)-L\left(\bar{u}_{1}, \bar{u}_{2}\right)\right\| \leq \frac{C A}{2 B}\left(\left\|u_{1}-\bar{u}_{1}\right\|_{L^{\infty}(0, A)}+\left\|u_{2}-\bar{u}_{2}\right\|_{L^{\infty}(0, A)}\right) .
$$

If $\frac{C A}{2 B}<1$, then the map $L$ admits a unique fixed point $\left(u_{1}^{*}, u_{2}^{*}\right)$, by the Banach Contraction Theorem. Next, we show that this fixed point is an optimal control pair, by using the minimizers, $\left(u_{1}^{\varepsilon}, u_{2}^{\varepsilon}\right)$, from 
Ekeland's Principle. To do this, we use the states $\left(x^{\varepsilon}, y^{\varepsilon}, V^{\varepsilon}, S^{\varepsilon}, i^{\varepsilon}\right)$ and adjoints $\left(\lambda^{\varepsilon}, \xi^{\varepsilon}, \eta^{\varepsilon}, p^{\varepsilon}, q^{\varepsilon}\right)$ corresponding to the minimizer $\left(u_{1}^{\varepsilon}, u_{2}^{\varepsilon}\right)$. Now, for

$K^{\varepsilon}(\tau)=\int_{0}^{T} i^{\varepsilon}(\tau, t) d t, a^{\varepsilon}(\tau)=\beta_{1} V^{\varepsilon}(\tau) x^{\varepsilon}(\tau)\left(\xi^{\varepsilon}(\tau)-\lambda^{\varepsilon}(\tau)\right)-\hat{\beta_{1}} V^{\varepsilon}(\tau) x^{\varepsilon}(\tau) \eta^{\varepsilon}(\tau)$ and $b^{\varepsilon}(\tau)=$ $\nu_{1} d_{1} \eta^{\varepsilon}(\tau) y^{\varepsilon}(\tau)$, we have

$$
\begin{aligned}
& \left\|L\left(u_{1}^{\varepsilon}, u_{2}^{\varepsilon}\right)-\mathcal{F}\left(\frac{a^{\varepsilon}-A_{2} K^{\varepsilon}-\sqrt{\varepsilon} \kappa_{1}^{\varepsilon}}{2 B}, \frac{b^{\varepsilon}-A_{3} K^{\varepsilon}-\sqrt{\varepsilon} \kappa_{2}^{\varepsilon}}{2 B}\right)\right\|_{\left(L^{\infty}(0, A)\right)^{2}} \\
= & \left\|\mathcal{F}\left(\frac{a^{\varepsilon}-A_{2} K^{\varepsilon}}{2 B}, \frac{b^{\varepsilon}-A_{3} K^{\varepsilon}}{2 B}\right)-\mathcal{F}\left(\frac{a^{\varepsilon}-A_{2} K^{\varepsilon}-\sqrt{\varepsilon} \kappa_{1}^{\varepsilon}}{2 B}, \frac{b^{\varepsilon}-A_{3} K^{\varepsilon}-\sqrt{\varepsilon} \kappa_{2}^{\varepsilon}}{2 B}\right)\right\|_{\left(L^{\infty}(0, A)\right)^{2}} \\
\leq & \left\|\frac{\sqrt{\varepsilon} \kappa_{1}^{\varepsilon}}{2 B}\right\|_{L^{\infty}(0, A)}+\left\|\frac{\sqrt{\varepsilon} \kappa_{2}^{\varepsilon}}{2 B}\right\|_{L^{\infty}(0, A)}=\frac{\sqrt{\varepsilon}}{B} .
\end{aligned}
$$

Now, we show that

$$
\left(u_{1}^{\varepsilon}, u_{2}^{\varepsilon}\right) \rightarrow\left(u_{1}^{*}, u_{2}^{*}\right) \quad \text { in } \quad L^{\infty}(0, A) \times L^{\infty}(0, A) .
$$

For $K^{*}(\tau)=\int_{0}^{T} i^{*}(\tau, t) d t$ and $K^{\varepsilon}(\tau)=\int_{0}^{T} i^{\varepsilon}(\tau, t) d t$, we have

$\left\|\left(u_{1}^{*}, u_{2}^{*}\right)-\left(u_{1}^{\varepsilon}, u_{2}^{\varepsilon}\right)\right\|_{\left(L^{\infty}(0, A)\right)^{2}}$

$$
\begin{aligned}
= & \left\|u_{1}^{*}-u_{1}^{\varepsilon}\right\|_{L^{\infty}(0, A)}+\left\|u_{2}^{*}-u_{2}^{\varepsilon}\right\|_{L^{\infty}(0, A)} \\
= & \left\|\mathcal{F}_{1}\left(\frac{\beta_{1} V^{*} x^{*}(\xi-\lambda)-\beta_{1} V^{*} x^{*} \eta-A_{2} K^{*}}{2 B}\right)-\mathcal{F}_{1}\left(\frac{a^{\varepsilon}-A_{2} K^{\varepsilon}-\sqrt{\varepsilon} \kappa_{1}^{*}}{2 B}\right)\right\|_{L^{\infty}(0, A)} \\
& +\left\|\mathcal{F}_{2}\left(\frac{\nu_{1} d_{1} y^{*} \eta-A_{3} K^{*}}{2 B}\right)-\mathcal{F}_{2}\left(\frac{\nu_{1} d_{1} y^{\varepsilon} \eta^{\varepsilon}-A_{3} K^{\varepsilon}-\sqrt{\varepsilon} \kappa_{2}^{\varepsilon}}{2 B}\right)\right\|_{L^{\infty}(0, A)} \\
\leq & \left\|L\left(u_{1}^{*}, u_{2}^{*}\right)-L\left(u_{1}^{\varepsilon}, u_{2}^{\varepsilon}\right)\right\|_{L^{\infty}(0, A)} \\
& +\left\|L\left(u_{1}^{\varepsilon}, u_{2}^{\varepsilon}\right)-\mathcal{F}\left(\frac{a^{\varepsilon}-A_{2} K^{\varepsilon}-\sqrt{\varepsilon} \kappa_{1}^{\varepsilon}}{2 B}, \frac{\nu_{1} d_{1} \eta^{\varepsilon} y^{\varepsilon}-A_{3} K^{\varepsilon}-\sqrt{\varepsilon} \kappa_{2}^{\varepsilon}}{2 B}\right)\right\|_{L^{\infty}(0, A)} \\
\leq & \frac{C A}{2 B}\left(\left\|u_{1}^{*}-u_{1}^{\varepsilon}\right\|_{L^{\infty}(0, A)}+\left\|u_{2}^{*}-u_{2}^{\varepsilon}\right\|_{L^{\infty}(0, A)}\right)+\frac{\sqrt{\varepsilon}}{B},
\end{aligned}
$$

from equations (3.51) and (3.52). Thus,

$$
\left\|u_{1}^{*}-u_{1}^{\varepsilon}\right\|_{L^{\infty}(0, A)}+\left\|u_{2}^{*}-u_{2}^{\varepsilon}\right\|_{L^{\infty}(0, A)} \leq \frac{C A}{2 B}\left(\left\|u_{1}^{*}-u_{1}^{\varepsilon}\right\|_{L^{\infty}(0, A)}+\left\|u_{2}^{*}-u_{2}^{\varepsilon}\right\|_{L^{\infty}(0, A)}\right)+\frac{\sqrt{\varepsilon}}{B} .
$$

Whence,

$$
\left\|u_{1}^{*}-u_{1}^{\varepsilon}\right\|_{L^{\infty}(0, A)}+\left\|u_{2}^{*}-u_{2}^{\varepsilon}\right\|_{L^{\infty}(0, A)} \leq \frac{\sqrt{\varepsilon} / B}{1-\frac{C A}{2 B}},
$$

for $A / 2 B$ sufficiently small. Equivalently,

$$
\left\|\left(u_{1}^{*}, u_{2}^{*}\right)-\left(u_{1}^{\varepsilon}, u_{2}^{\varepsilon}\right)\right\|_{L^{\infty}(0, A) \times L^{\infty}(0, A)} \leq \frac{\sqrt{\varepsilon} / B}{1-\frac{C A}{2 B}} \rightarrow 0 \quad \text { as } \quad \varepsilon \rightarrow 0^{+} .
$$

Thus,

$$
\left(u_{1}^{\varepsilon}, u_{2}^{\varepsilon}\right) \rightarrow\left(u_{1}^{*}, u_{2}^{*}\right) \quad \text { in } \quad L^{\infty}(0, A) \times L^{\infty}(0, A) .
$$

Finally, we show that $\left(u_{1}^{*}, u_{2}^{*}\right)$ is the minimizer of the functional, $\mathcal{J}$. Now, as the functional, $\mathcal{J}$, is lower semicontinuous, using Ekeland's Principle, we have

$\mathcal{J}\left(u_{1}^{\varepsilon}, u_{2}^{\varepsilon}\right) \leq \inf _{\left(u_{1}, u_{2}\right) \in \mathcal{U}} \mathcal{J}\left(u_{1}, u_{2}\right)+\varepsilon$. Since $\left(u_{1}^{\varepsilon}, u_{2}^{\varepsilon}\right) \rightarrow\left(u_{1}^{*}, u_{2}^{*}\right)$ as $\varepsilon \rightarrow 0^{+}$, it follows that $\mathcal{J}\left(u_{1}^{*}, u_{2}^{*}\right) \leq$ $\inf _{\left(u_{1}, u_{2}\right) \in \mathcal{U}} \mathcal{J}\left(u_{1}, u_{2}\right)$. 


\section{Numerical Simulations}

We present a numerical scheme for the within-host model (2.1)-(2.4) and between-host model (2.5)-(2.7) based on semi-implicit finite-difference schemes for ordinary differential equations $[24,26]$ and partial differential equations [2, p. 166]. Let $\Delta \tau=h>0$ be the discretization step for the interval $[0, A]$, with $h=\frac{A}{M}$, where $M$ is the total number of subintervals in age (age-since-infection), and $\Delta t=k>0$ be the discretization step for the interval $[0, T]$, with $k=\frac{T}{N}$, where $N$ is the total number of subintervals in time. We discretize the intervals $[0, A]$ and $[0, T]$ at the points $\tau_{j}=j \Delta \tau(j=0,1, \ldots, M)$ and $t_{n}=n \Delta t(n=0,1, \ldots, N)$, respectively. Next, we define the state and adjoint functions $x, y, V, S, \omega($ where $\omega \equiv i), \lambda, \xi, \eta, p, q$, and controls $u_{1}$ and $u_{2}$ in terms of nodal points $x^{j}, y^{j}, V^{j}, S^{n}, w_{j}^{n}, \lambda^{j}, \xi^{j}, \eta^{j}$, $p^{n}, q_{j}^{n}, u_{1}^{j}$ and $u_{2}^{j}$. Since $\omega_{j}^{n}$ is an approximation to the solution of the equation that models infectious individuals at time level $t_{n}$ and grid point $\tau_{j}$, we approximate the directional derivatives $\frac{\partial \omega(\tau, t)}{\partial t}$ and $\frac{\partial \omega(\tau, t)}{\partial \tau}$ by

$$
\frac{\partial \omega\left(\tau_{j}, t_{n}\right)}{\partial t} \approx \frac{\omega_{j}^{n}-\omega_{j}^{n-1}}{\Delta t} \text { and } \frac{\partial \omega\left(\tau_{j}, t_{n}\right)}{\partial \tau} \approx \frac{\omega_{j}^{n-1}-\omega_{j-1}^{n-1}}{\Delta \tau} .
$$

Age of individuals changes at the same speed as chronological time, and therefore we assume that $\Delta t=$ $\Delta \tau$, so that

$$
\frac{\partial \omega\left(\tau_{j}, t_{n}\right)}{\partial t}+\frac{\partial \omega\left(\tau_{j}, t_{n}\right)}{\partial \tau} \approx \frac{\omega_{j}^{n}-\omega_{j-1}^{n-1}}{\Delta t}
$$

Since initial conditions are given for the state system, we use the forward finite-difference approximation to obtain a semi-implicit scheme for the state system. Similarly, since final time conditions of the adjoint system are given, we approximate the time-since-start of infection, chronological time and age-sinceinfection derivatives of the adjoint functions by their first-order semi-implicit backward finite-difference approximations. To fully implement our numerical scheme for the coupled model, we use the parameter values of the within-host and epidemiological model of HIV given in Table 3, and the forward-backward sweep method, whereby solutions to the state system are obtained using a finite difference forward sweep method and solutions to the adjoint system are obtained using a finite difference backward sweep method [39]. We now illustrate numerical simulations of the optimal control and corresponding states for one sample set of parameters. For this set of parameters without control, we have $\mathcal{R}_{0}=4.3$.

Table 3: Within-Host Model Parameter Values

\begin{tabular}{|l|l|l|}
\hline Parameter & Value & Source \\
\hline$r$ & $10{\text { cells } \mathrm{mm}^{-3} \text { day }^{-1}}^{-1}$ & {$[9,18,26,38,44,49]$} \\
\hline$\mu$ & 0.02 day $^{-1}$ & {$[9,26,44,49]$} \\
\hline$\beta_{1}$ & $2.4 \times 10^{-5} \mathrm{~mm}^{3}$ day $^{-1}$ & {$[9,18,26,38,44,49]$} \\
\hline$\hat{\beta}_{1}$ & $2.4 \times 10^{-5} \mathrm{~mm}^{3}$ day $^{-1}$ & {$[9,18,26,38,44,49]$} \\
\hline$d_{1}$ & 0.5 day $^{-1}$ & {$[9,18,26,38,44]$} \\
\hline$\nu_{1}$ & 1200 virions cell $^{-1}$ & {$[18]$} \\
\hline$\delta_{1}$ & 3 day $^{-1}$ & {$[9,18,44]$} \\
\hline$s_{1}$ & 1.4 day $^{-1}$ & assumed \\
\hline$c_{1}$ & $4 \times 10^{-5} \mathrm{~mm}^{3}$ virion $^{-1}$ year $^{-1}$ & assumed \\
\hline$\mu_{1}$ & $2 \times 10^{-7}$ virion $^{-1}$ year $^{-1}$ & assumed \\
\hline$m_{0}$ & $0.012 \mathrm{~mm}^{3}$ year $^{-1}$ & assumed \\
\hline$\Lambda$ & 2750 humans $^{2}$ & assumed \\
\hline
\end{tabular}

In Figure 1, we have trajectories representing healthy $\mathrm{CD} 4^{+} \mathrm{T}$ cells, infected $\mathrm{CD} 4^{+} \mathrm{T}$ cells and free virus in the absence/presence of transmission and virion production suppressing drugs for a total of 100 days. In the absence of drugs and starting with 600 healthy $\mathrm{CD} 4^{+} \mathrm{T}$ cells per $\mathrm{mm}^{3}$ of blood, the number of heathy cells decreases greatly within the first 20 days of infection. Between $20-100$ days, the count of 
$\mathrm{CD}^{+}$healthy cells lies below 200 . With no infected $\mathrm{CD} 4^{+} \mathrm{T}$ cells in the population at the beginning of the infection, the number of infected cells increases significantly between 10 - 30 days, with a maximum count of about 190 infected cells, and decreases thereof. Starting with 0.005 virions per $\mathrm{mm}^{3}$ of blood, an acute phase is observed between 10 - 30 days since start-of-infection with a maximum count of about $2.5 \times 10^{4}$ virions, followed by a latent period.
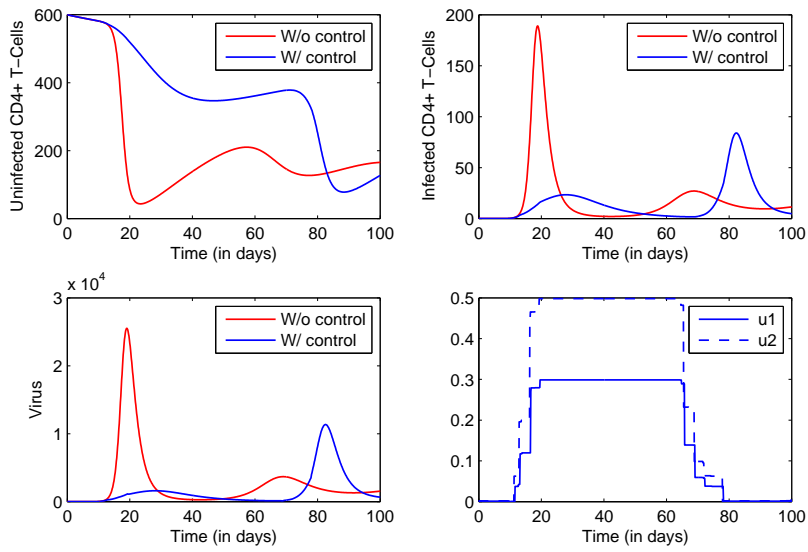

Figure 1: Healthy CD4 ${ }^{+}$T Cells, Infected CD $4^{+} \mathrm{T}$ Cells, Free Virus with and without Control when $x^{0}=600$ cells $\mathrm{mm}^{-3}, y^{0}=0$ cell $\mathrm{mm}^{-3}, V^{0}=0.005$ virions $\mathrm{mm}^{-3}, A_{1}=1, A_{2}=0.7, A_{3}=0.7, \mathrm{~A}=100$ days and $B=5 \times 10^{6}$.

In the presence of transmission and virion production suppressing drugs, trajectories indicate an increase in the number of healthy $\mathrm{CD} 4^{+} \mathrm{T}$ cells, and a decrease in infected CD4 ${ }^{+} \mathrm{T}$ cells and free virus. Also, the acute phase observed in the virus population within 10 - 30 days occurs with lower severity, and the viral relapse phase in the absence of control occurs sooner than in the presence of control. Similarly, the acute phase observed in the population of infected $C D 4^{+}$T-cells within $10-30$ days occurs with lower severity. The control program suggests full treatment between 10 - 80 days since start-of-infection.

Figure 2 represents the between-host dynamics in the absence/presence of transmission and virion production transmission suppressing drugs. In the absence of drugs, trajectories for susceptible individuals suggest a steady decrease in the population of susceptible individuals at the epidemiological level as the result of the proliferation of free virus at the within-host level. Also, with the assumption that at time $t=0$, the initial age distribution of infectious individuals is modeled by $i(\tau, 0)=100 \sin \left(\frac{\pi \tau}{25}\right)$, we observe an oscillatory increase in the number of infectious individuals in the population as time evolves. In the presence of transmission and virion production suppressing drugs, there are more susceptibles with a lower prevalence rate.

Our numerical results suggest that at the within-host level, the acute phase of infection observed within $2-4$ weeks occurs with lower severity, followed by a latent phase between $4-10$ weeks. During week 11 , the virus proliferates, with a less severe effect relative to the population of free virus in the absence of control. Moreover, when transmission and virion production suppressing drugs are administered, the susceptible population experiences an increase while the infectious population experiences a significant decrease in prevalence. 

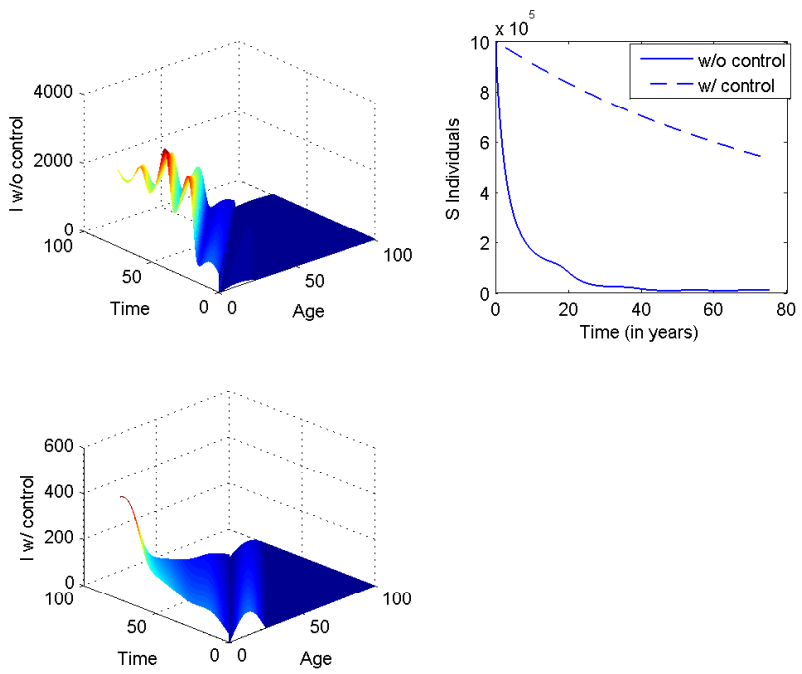

Figure 2: Susceptible and Infectious Individuals with and without Control, and Initial Age Distribution $i(\tau, 0)=$ $100 \sin \left(\frac{\pi \tau}{25}\right)$ when $\Lambda=2750, x^{0}=600$ cells per $\mathrm{mm}^{3}, y^{0}=0$ cell per $\mathrm{mm}^{3}$ and $V^{0}=0.005$ virions per $\mathrm{mm}^{3}$.

\section{Conclusion}

We formulated, in a careful way, a within-host model linked with an epidemiological model through a structural variable and coefficients. Existence and uniqueness results of the epidemiological model are established. Then we derived an explicit expression for the basic reproduction number of the epidemiological model, using the next generation method and examined conditions for existence of an endemic equilibrium. We showed that the disease-free equilibrium is locally asymptotically when $\mathcal{R}_{0}<1$ and unstable if $\mathcal{R}_{0}>1$. Also, when $\mathcal{R}_{0}<1$, the disease-free equilibrium is globally stable. If $\mathcal{R}_{0}>1$, we showed that there exists an endemic equilibrium which is locally asymptotically stable when the maximal age of infection, $A$, is large enough.

We formulated an optimal control problem which aims at minimizing infectious individuals, free virus and toxicity cost. In order to curtail the proliferation of the virus at the within-host level, we incorporated transmission and virion production suppressing drugs into the the within-host model. We establish a Lipschitz property for the within-host and between-host state solutions in terms of functions representing transmission and virion production suppressing drugs. We derived sensitivity equations for the coupled model which were used in deriving an adjoint system. We obtained an optimal control characterization for the control pair and established the existence of optimal control using Ekeland's Principle. Using a minimizing sequence obtained via Ekeland's Principle, we established uniqueness results.

Using a forward-backward finite difference approximation, we solved the optimality system numerically for illustration. In the absence of control in the population, numerical simulations indicate a decrease in the number of healthy $\mathrm{CD} 4{ }^{+}$cells, and an increase in the number of infected cells and free virus within the first few days of infection at the within-host level. At the between-host level, there is a sustained decrease in the number of susceptible individuals and an oscillatory increase in the number of infectious cases. In the presence of transmission and virion production suppressing drugs, more healthy cells were observed with fewer infected cells and free virus at the within-host level. Also, fewer infectious cases were observed with a significant increase in the population of susceptible humans in the presence of transmission and virion production suppressing drugs. Investigation of numerical results when varying other parameters should be considered in the future. 
We developed novel optimal control results for our linked system. Our analysis and control techniques give a new tool for investigating immuno-epidemiological models for other diseases.

Acknowledgements. Numfor and Lenhart acknowledge partial support from the National Institute for Mathematical and Biological Synthesis through the National Science Foundation Award

\# EF-0832858, and Lenhart is also partially supported by the University of Tennessee Center for Business and Economic Research.

\section{References}

[1] R. M. Anderson, R. M. May. the Invasion, Persistence and Spread of Infectious Diseases Within Animal and Plant Communities. Phil. Trans. R. Soc. Lond., 314 (1986), 533-570.

[2] S. Anita, V. Arnăutu, V. Capasso. An Introduction to Optimal Control Problems in Life Sciences. Springer Science, New York, 2011.

[3] S. Anita. Analysis and Control of Age-Dependent Population Dynamics. Kluwer Academic Publishers, Dordretcht, 2000.

[4] S. Anita. Optimal Control Harvesting for a Nonlinear Age-dependent Population Dynamics. J. Math. Anal. Appl., 226 (1998), 6-22.

[5] R. Antia, B. Levin, R. M. May. Witin-host Population Dynamics and the Evolution and Maintenance of Microparasite Virulence. Am. Nat., 144 (1994), 457-472.

[6] V. Barbu, M. Iannelli. Optimal Control of Population Dynamics. J. Optim. Theory Appl., 102 (1999),1-14.

[7] V. Barbu. Mathematical Methods in Optimization of Differential Systems. Kluwer Academic Publishers, Dordretcht, 1994.

[8] M. Brokate. Pontryagin's Principle for Control Problems in Age-Dependent Population Dynamics. J. Math. Biol., 23 (1985), 75-101.

[9] S. Butler, D. Kirschner, S. Lenhart. Optimal Control of Chemotherapy Affecting the Infectivity Of HIV, Advances In Mathematical Population Dynamics-Molecules, Cells And Man. Editors: O. Arino, D. Axelrod, And M. Kimmel, World Scientific Press, Singapore, (1997) 557-569.

[10] C. Castillo-Chavez, Z. Feng. Global Stability of an Age-Structured Model for TB and its Applications to Optimal Vaccination Strategies. Math.Biosci., 151 (1998), 135-154.

[11] D. Coombs, M. A. Gilchrist, J. Percus, A. S. Perelson. Optimizing Viral Production. Bull. Math. Biol., 65 (2003) 1003-1023.

[12] U. Dissanayake, S. Dias, H. Polson, S. Longacre, P. Udagama-Randeniya. Immuno-Epidemiology of Plasmodium Vivax Merozoite Surface Proten-4. Bio. Sci., 37 (2008) 97-105.

[13] I. Ekeland. On the Variational Principle. J. Math. Appl., 47 (1974), 324-353.

[14] L. C. Evans. Partial Differential Equations. American Mathematical Society, 2nd edition, 2010.

[15] L. C. Evans, R. F. Gariepy. Measure Theory and Fine Properties of Functions. CRC Press, Boca Raton, 1992.

[16] Z. Feng, J. Velasco-Hernandez, B. Tapia-Santos. A Mathematical model for Coupling within-Host and Between-Host Dynamics in an Environmentally-driven Infectious Disease. Math. Biosci., 241 (2013), 49-55.

[17] Z. Feng, J. Velasco-Hernandez, B. Tapia-Santos, M. C. Leite. A Model for Coupled Within-Host and Between-Host Dynamics in an Infectious Disease. Nonlinear Dyn., 68 (2012), 401-411.

[18] K. R. Fister, S. Lenhart, J. S. McNally. Optimizing Chemotherapy in an HIV Model. J. of Differential Equations, 1998 (1998), 1-12.

[19] K. R. Fister, S. Lenhart. Optimal Harvesting in an Age-Structured Predator-Prey Model. Appl. Math. Optim., 54 (2006), 1-15.

[20] K. R. Fister, S. Lenhart. Optimal Control of a Competitive System with Age-Structured. J. Math. Anal., 291 (2004), $526-537$.

[21] V. V. Ganusov, C. T. Bergstrom, R. Antia. Within-Host Population Dynamics and the Evolution of Microparasites in a Heterogeneous Host Population. Evolution, 56 (2002), no. 2, 213-223.

[22] M. A. Gilchrist, D. A. Coombs. Evolution of Virulence: Interdependence, Constraints, and Selection using Nested models. Theor. Pop. Biol., 69 (2006), 145-153.

[23] M. A. Gilchrist, A. Sasaki. Modeling Host-Parasite Coevolution: A Nested Approach based on Mechanistic Models. J. Theor. Biol., 218 (2002), 289-308.

[24] A. B. Gumel, P. N. Shivakumar, B. M. Sahai. A Mathematical Model for the Dynamics of HIV-1 During the Typical Course of Infection. Nonlinear. Anal., 47 (2001), 1773-1783.

[25] M. E. Gurtin, R. C. MacCamy. Nonlinear Age-dependent Population Dynamics. Arch. Rat. Mech. Anal., 54 (1974), 281-300.

[26] K. Hattaf, N. Yousfi. Two optimal Treatments of HIV Infection Model. World J. Modell. Simul., 8 (2012), $27-35$.

[27] J. M. Heffernan. Mathematical Immunology of Infectious Diseases. Math. Popul. Stud., 18 (2011), 47-54.

[28] J. M. Heffernan, R. J. Smith, L. M. Wahl. Perspectives on Basic Reproduction Ratio. J. R. Soc. Interface, 2 (2005), 281-293. 
[29] B. Hellriegel. Immunoepidemiology-Bridging the Gap Between Immunology and Epidemiology. TRENDS in Parasitology, 17 (2001), 102-106.

[30] Y. Hsieh, S. Sheu. The Effect of Density-Dependent Treatment and Behaviour change on the Dynamics of HIV Transmission. J. Math. Biol., 43 (2001), 69-80.

[31] M. Iannelli, M. Martcheva, F. A. Milner. Gender-Structured Population Modeling: Mathematical Methods. Numerics, and Simulations, SIAM, Philadelphia, 2005.

[32] H. Joshi. Optimal Control of an HIV Immunology Model. Optimal Control Appl. Methods, 23 (2002), $199-213$.

[33] H. Joshi, S. Lenhart, K. Albright, K. Gipson. Modeling the Effect of Information Campaign on the HIV Epidemic in Uganda, Math. Biosci. Eng., 5 (2008), 757-770.

[34] H. Joshi, S. Lenhart, M. Y. Li, L. Wang. Optimal Control Methods Applied to Disease Models. Contemp. Math., 410 (2006), 187-207.

[35] M. Kgosimore, E. M. Lungu. The Effects of Vertical Transmission on the Spread of HIV/AIDS in the Presence of Treatment. Math. Biosci. Eng., 3 (2006), 297-312.

[36] D. Kirschner. Using Mathematics to Understand HIV Immune Dynamics. AMS Notices, 432 (1996), $191-202$.

[37] D. Kirschner, G. F. Webb. A Model for Treatment Strategy in the Chemotherapy of AIDS. Bull. Math.Biol., 58 (1996), $367-391$.

[38] D. Kirschner, S. Lenhart, S. Serbin. Optimal Control of the Chemotherapy of HIV. J. Math. Biol., 35 (1997), $775-792$.

[39] S. Lenhart, J. T. Wortman. Optimal Control Applied to Biological Models. Taylor \& Francis, Boca Raton, FL, 2007.

[40] X. Li, J. Liu, M. Martcheva. An Age-Structured Two-strain Epidemic Model with Superinfection. Math. Biosci. Eng., 7 (2010), 123-147.

[41] M. Martcheva. An Immuno-epidemiological Model of Paratuberculosis. AIP Conf. Proc., 1404 (2011), $176-183$.

[42] M. Martcheva, F. A. Milner. A Two-Sex Age-Structured Population Model: Well Posedness. Math. Population Studies, 7 (1999), 111-129.

[43] L. F. Murphy, S. J. Smith. Optimal Harvesting of an Age-Structured Population. J. Math. Biol., 29 (1990), 77-90 .

[44] A. Perelson, D. E. Kirschner, R. D. Boer. Dynamics of HIV Infection of CD4 ${ }^{+}$T Cells. Math. Biosci., 114 (1993), 81-125.

[45] Z. Qiu, X. Li, M. Martcheva. Multi-strain Persistence Induced by Host Age Structure. J. Math. Anal. Appl., 391 (2012), 395-612.

[46] E. Shim, Z. Feng, M. Martcheva. C. Castillo-Chavez, An Age-Structured Epidemic Model of Rotavirus with Vaccination. Math. Biol., 53 (2006), 719-746.

[47] R. J. Smith. Explicitly Accounting for Antiretroviral Drug Uptake in Theoretical HIV Models Predicts Long-term Failure of Protease-only Therapy. J. Theor Biol., 251 (2008), 227-237.

[48] G. F. Webb, Theory of Nonlinear Age-Dependent Population Dynamics, Marcel Dekker, Inc, New York, 1985.

[49] E. K. Yeargers, R. W. Shonkwiler, J. V. Herod, An Introduction to the Mathematics of Biology with Computer Algebra Models, Birkhäuser, Boston, 1996. 